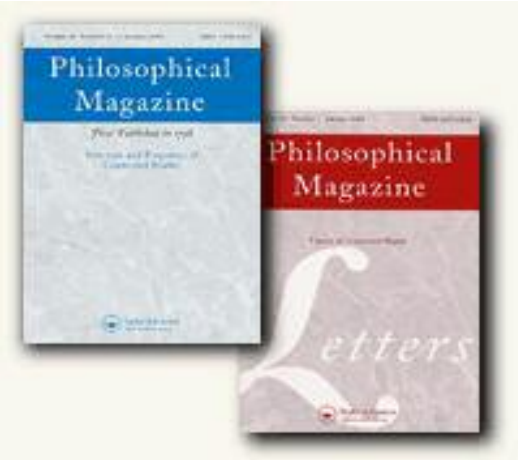

\title{
Magnetism in Quasicrystals
}

\begin{tabular}{|r|l|}
\hline Journal: & Philosophical Magazine \& Philosophical Magazine Letters \\
\hline Manuscript ID: & TPHM-07-Oct-0291.R1 \\
\hline Journal Selection: & Philosophical Magazine \\
\hline Date Submitted by the & 10 -Jan-2008 \\
\hline Complete List of Authors: & $\begin{array}{l}\text { HIPPERT, Francoise; Laboratoire des Materiaux et du Genie } \\
\text { Physique, INP-Grenoble } \\
\text { PREJEAN, Jean-Jacques; CNRS, Institut Neel }\end{array}$ \\
\hline Keywords: & $\begin{array}{l}\text { magnetic structure, magnetic transitions, magnetism, quasicrystals, } \\
\text { transport properties }\end{array}$ \\
\hline Keywords (user supplied): & magnetic structure, magnetic transitions, magnetism \\
\hline \multicolumn{2}{|l|}{$\begin{array}{l}\text { Note: The following files were submitted by the author for peer review, but cannot be converted } \\
\text { to PDF. You must view these files (e.g. movies) online. }\end{array}$} \\
\hline Phil-Mag-eps.tex
\end{tabular}

\section{S) ScholaroNE \\ Manuscript Central}




\title{
Magnetism in Quasicrystals
}

\author{
F. HIPPERT * $†$ and J.J PREJEAN †† \\ $\nmid$ Laboratoire des Matériaux et du Génie Physique, INP-Grenoble, 38016 Grenoble, France
}

††Institut Neel, CNRS, BP 166, 38042 Grenoble, France

(version)

\begin{abstract}
The present understanding of magnetic properties of quasicrystals (QCs) is reviewed. In AlMn and AlPdMn QCs, only a few $M n$ atoms, with concentration from a few $\%$ down to $10^{-4}$ carry a localized magnetic moment. These moments are coupled via RKKY interactions, leading to a canonical spin glass ordering at low temperature. They also exhibit a Kondo effect and $A l P d M n$ QCs turned out to be a model system for studying experimentally the competition between Kondo and RKKY interactions in the absence of long range magnetic ordering. Besides, the presence of localized moments has very unusual consequences on the electronic transport in AlPdMn QCs. In $R M g Z n$ and $R M g C d$ QCs ( $R$ is a magnetic rare earth), with $R$ concentration around $9 \%$, a spin glass like freezing is observed. However the short range antiferromagnetic correlations detected above the freezing temperature are unusual and certainly influenced by the quasiperiodicity.
\end{abstract}

\section{Introduction}

Magnetic moments only exist in a minority of the numerous discovered QCs. Nevertheless magnetism in quasicrystals is a fascinating field of investigation, obviously in the case of $R M g Z n$ QCs with $R$ a magnetic rare earth, but also in the case of $A l$-based QCs containing $M n$. In many QCs, such as AlLiCu, GaMgZn, ..., the absence of magnetic moments is a trivial observation since none of their constituting elements is expected to be magnetic. The case of $A l$-based QCs with transition metals was more striking. The $N i$ and $C o$ are found non magnetic ${ }^{1}$, which suggests that the electronic structure of QCs does not favor moment formation. But $M n$ localized moments have been systematically detected since the pioneering work of Hauser et al [1] published in 1986, very soon after the discovery of the first QCs in the AlMn system [2]. The magnetic susceptibility $\chi$, deduced from the linear response of the magnetization to a small static field, was found to obey a Curie-Weiss law: $\chi \approx C /(T+\theta)$ above $5 \mathrm{~K}$ in icosahedral AlMn and AlSiMn QCs with $M n$ content from 14 to $22 \%$, which proves the existence of local moments. A peak in the $\chi_{a c}(T)$ obtained using a small ac field (frequency $\approx$ a few $\mathrm{Hz}$ ) was also observed at a temperature $T_{f}<5 \mathrm{~K}$. This proves the presence of magnetic interactions between the local moments. A spin glass behavior was proposed. A quite intriguing fact was the weakness of the Curie constant $C$, found much smaller than calculated if all the $M n$ atoms are magnetic, each carrying a spin $S$ close to $5 / 2$. The Curie constant is proportional to $N x S(S+1)$ with $N$ the total number of atoms and $x$ the concentration of local moments. Soon after, in another pioneering work [3], Warren et al demonstrated by observing a ${ }^{55} \mathrm{Mn}$ nuclear magnetic resonance (NMR) that most of the $M n$ sites do not carry a local moment, which should explain the small Curie constant. Subsequently, the same features (small Curie terms, non-magnetic $M n$ and $\chi_{a c}$ peak) were observed in the successively discovered QC phases: The decagonal metastable AlMn [4], the stable icosahedral AlPdMn ( $[5,6]$ and references therein) and also the decagonal AlPdMn phases $[7,8]$. Recently magnetic moments have been observed in $Z n M S c$ QCs [9] with $M=M n$ and $\mathrm{Fe}^{2}, \mathrm{Co}$ and $\mathrm{Ni}$

\footnotetext{
*Corresponding author. Email: francoise.hippert@inpg.fr

${ }^{1}$ Very small and non reproducible Curie-like terms may be observed in the susceptibility $(\chi=C / T)$ at low temperature. Their magnitude being the same as that of unavoidable magnetic impurities, their study is of little significance. Besides, ferromagnetic contributions (with Curie temperature much above the room temperature) are often detected in $A l$-based QCs. They are ascribed to foreign phases (probably surface phases) non detected in $\mathrm{X}$ ray diffraction.

${ }^{2}$ This is the first observation of a significant Fe magnetism in QCs up to now and this deserves further investigations. In the case of $A l C u F e$, localized moments are indeed detected [10] but their concentration remains very small whatever the alloy composition, in strong
}

Philosophical Magazine

ISSN 1478-6435 print/ISSN 1478-6443 online (C) 200x Taylor \& Francis

http://www.tandf.co.uk/journals

DOI: $10.1080 / 1478643 Y Y x x x x x x x x$ 
being found non magnetic.

Magnetism of $M n$ in $A l$-based QCs motivated numerous experimental and theoretical works in order to answer several fundamental questions. The first series of questions concerns the occurrence of magnetism on a $M n$ site: First of all, why most of the $M n$ atoms are non magnetic? Which is the spin value of the magnetic $M n$ ? Are the latter located in some foreign magnetic phases? If they belong to the QC phase, are they located on specific sites, intrinsic to the structure, or is the moment formation induced by a local defect in the quasiperiodic potential? At the present time, it is possible to draw several major conclusions from systematic experimental studies of $A l M n$ and $A l P d M n$ QCs. The $M n$ atoms which carry a moment have a large spin value: $S \approx 5 / 2$. They do belong to the QC phase and their concentration is very low, from a few $\%$ down to $10^{-4}$. They are presumably randomly distributed in the quasiperiodic structure and their concentration depends drastically on the alloy composition. Thus the presence of a moment on a $M n$ site could originate in a neighboring local defect in the quasiperiodic structure. These results have motivated theoretical studies aimed at understanding the parameters governing moment formation on $M n$ atoms in $\mathrm{Al}$-based QCs and related crystalline phases. Another series of questions concern the nature of the magnetic interactions between the few $M n$ moments and that of the magnetic state below $T_{f}$. First, from the above results, one concludes that the magnetic moments do not form a quasiperiodic array. So it is not a surprise that the magnetic order is not specific to the QC structure: A standard spin glass transition is found to occur at $T_{f}$. It originates in RKKY (Ruderman-Kittel-Kasuya-Yosida) interactions, i.e. mediated by the polarization of the spins of the conduction electrons. The interaction between two moments alternates in sign rapidly with the relative distance $r$ of the two moments. Thus it can be either ferromagnetic (F) or antiferromagnetic (AF) according to the $r$ value. This feature associated to a random spatial distribution of the moments are the key ingredients to form a spin glass state [11], such as that one observed in dilute $C u M n$ alloys where a few $M n$ atoms (then all magnetic) are randomly distributed in a periodic $C u$ matrix. All these points will be developed in Section 2. It could not be imagined at the beginning that these studies would have fruitful consequences in other fields. Indeed, they allowed a better understanding of the unconventional transport properties of quasicrystals (Section 3). Besides, AlPdMn QCs provided the rare opportunity to allow a comprehensive study of the Kondo and RKKY interactions, which is a fundamental problem in magnetism, independently of the QC study (Section 4).

If the disordered repartition of magnetic $M n$ in Al-based QCs does not allow the existence of a specific magnetic order, a quite different situation occurs in the QCs containing magnetic rare earth $R$ atoms, either in the $R M g Z n$ or in the $R M g C d$ system. Since all the $R$ atoms are magnetic, these QCs allow to investigate the nature of magnetic correlations on a quasiperiodic lattice (section 5), a fundamental issue in the domain of magnetism in QCs [12]. Up to now only short-range correlations have been observed [13], the exact nature of the spin glass like transition $[14,15]$ remaining a debated question.

\section{$2 \quad M n$ magnetism in Al-based QC}

Fig. 1 illustrates two main features exhibited by all the $A l$-based QCs containing $M n$ : An ac-susceptibility peak and a Curie-like temperature dependence of $\chi(T)$ at $T>T_{f}{ }^{1}$. All these phases exhibit a very small Curie constant and a behavior at low temperature which suggests the occurrence of a spin glass state: For instance, a broad bump of the specific heat at a temperature around $T_{f},[4,6,16-18]$ and magnetic hysteresis features at $T<T_{f}[17,19]$. These observations were important but could not help to know the concentration of $M n$ atoms which are magnetic, their location, their spin value or the existence of a true spin glass transition. In order to clear up these issues, additional works had to be performed, as presented below.

contrast with the case of $A l P d M n$ and $A l M n$ QCs.

${ }^{1}$ Actually, the Curie like term is superposed to a negative temperature independent susceptibility $\chi_{0}$ which has been subtracted from the data in all the present figures. This negative $\chi_{0}$, reminiscent of the behaviour of non magnetic QCs, results from a weak (positive) Pauli susceptibility for the conduction electrons due to the reduced density of states at the Fermi level $N\left(E_{F}\right)$ (pseudo-gap) characteristic of QCs. Then the diamagnetic negative orbital contributions of ion cores and conduction electrons dominate. 

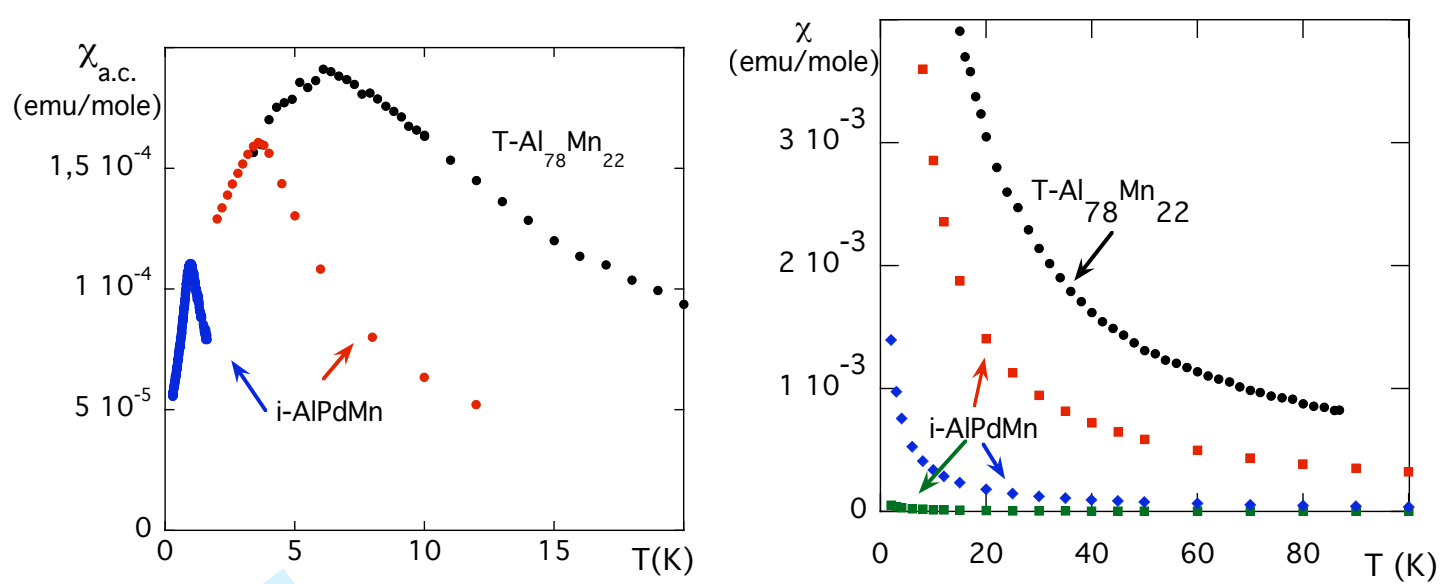

Figure 1. Susceptibility vs. temperature for several $A l M n$ and $A l P d M n$ QCs. Left: ac-susceptibility at low temperature showing a peak at $T_{f}$; Right: dc-susceptibility above $T_{f}$.

\subsection{Spin glass transition and deduction of the Mn spin value}

It is the thorough knowledge of the spin glass properties, acquired well before the QC discovery $[11,20]$, which helped to clarify the nature of the $M n$ magnetism in the $A l$-based QCs. Many features often claimed to be characteristic of spin glasses can be indeed observed in systems which are not spin glasses. For instance, small ferromagnetic particles (iron grains dispersed in an alumina matrix) exhibit precisely such features [21]: A sharp maximum in the $\chi_{a c}$ at a temperature $T_{f}$, the frequency dependence of $T_{f}$ observed in spin glasses and a magnetization below $T_{f}$ depending on time logarithmically and on the magneto-thermal history. The magnetic hysteresis was even shown to exist sizably in a region of the $(H, T)$-plane limited by a line obeying the law proposed theoretically by de Almeida and Thouless for mean field Ising spin glasses [11]. For this system, the above properties originate in a large distribution of blocking temperatures, while the Curie-Weiss temperature $\theta$ reflects the role of the dipolar interactions between the grain moments. So, to differentiate a spin glass from the latter system requires more elaborate studies. The more convincing one is the study of the temperature dependence of the so-called non linear susceptibilities above $T_{f}$, i.e. of the temperature dependence of the $A_{i}$ s involved in the series expansion of the magnetization $M(H)$ in terms of odd powers of $H$ :

$$
M(H, T)=C \times \frac{H}{T+\theta}-A_{3} \times\left[\frac{H}{T+\theta}\right]^{3}+A_{5} \times\left[\frac{H}{T+\theta}\right]^{5}-\ldots
$$

In spin glasses, $\theta \ll T_{f}$ in the temperature decade above $T_{f}$. This originates in some compensation of the effects of the $\mathrm{F}$ and $\mathrm{AF}$ interactions ( $\theta=0$ corresponds to an equal weight of these interactions). The interaction effects manifest themselves clearly in the terms non linear in field in Eq.1. A true phase transition is characterized by the divergence of a correlation length $\xi(T)$ at a temperature $T_{c}$. In the spin glass case, this results in a divergence of $A_{3}(T), A_{5}(T), \ldots$, which obey power laws of $\xi(T)$ [11]. In contrast, temperature independent $A_{i}$ s indicate the lack of divergence of any $\xi(T)$, which is the case for a collection of fine magnetic grains [21]. So the goal is to determine experimentally the $A_{i}$ values. The principle of the experimental procedure is simple. At each temperature $T_{i}$ (from high temperature down to $T_{f}$ ), $M(H$ ) is measured, which allows to deduce the prefactor of $H$ (i.e. the linear susceptibility $\chi$ ) and those of $H^{3}$, $H^{5}$ in the series expansion of $M$ (Eq. 1). (The measurement fields have to be sufficiently low so that the subsequent $H^{2 n+1}$ terms are negligible.) This allows to calculate the values of $A_{3}\left(T_{i}\right)$ and $A_{5}\left(T_{i}\right)$ once the $\theta$ value has been deduced from the temperature dependence of $\chi$. Actually, the study of $A_{3}$ and $A_{5}$ is difficult since one has to measure the very initial deviations of $M$ from its dominant term $\chi \mathrm{H}$. In practice, this requires an unusual relative precision (much better than $10^{-3}$ ) on $M, H, T$ at low temperature and in weak field conditions [20,22]. The work [22] performed on a melt spun $i-A l_{73} M n_{21} S i_{6}$ QC sample (for which $\theta=0$ ) showed that $A_{3}(T)$ and $A_{5}(T)$ vary by orders of magnitude when the temperature is 

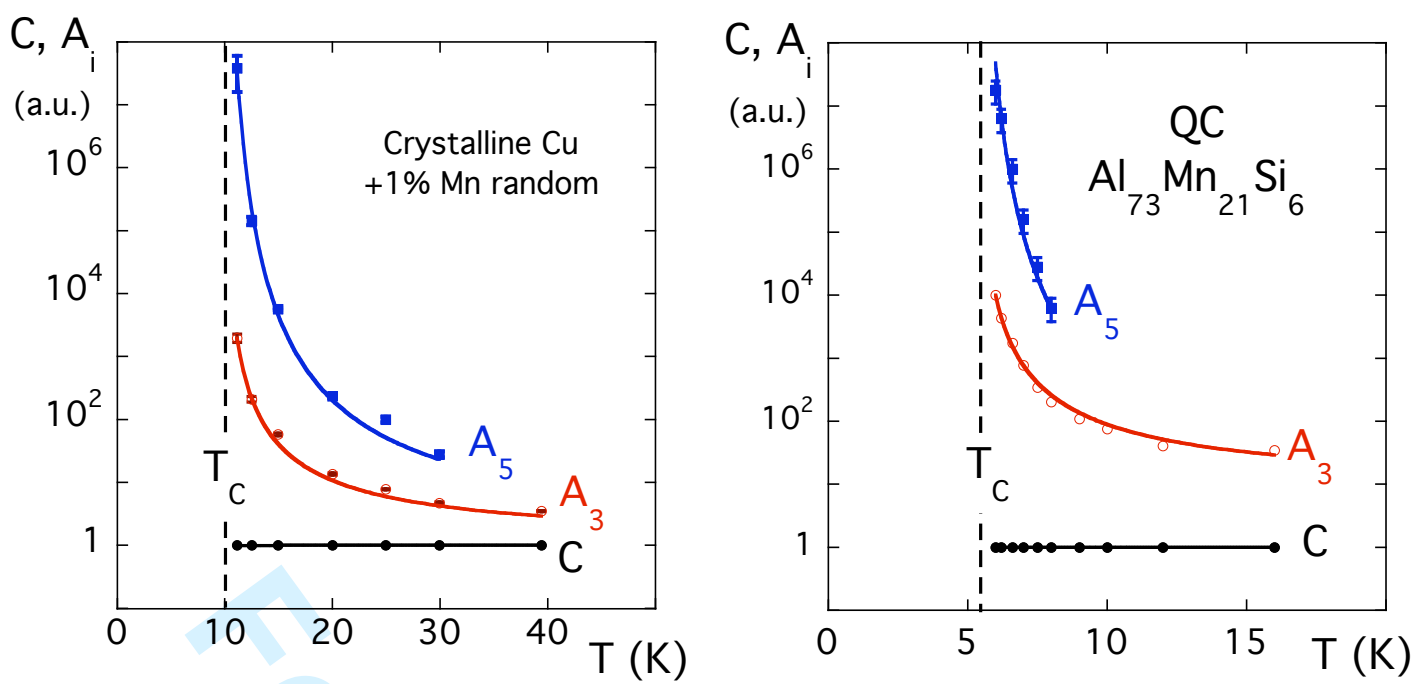

Figure 2. Temperature dependence of $C, A_{3}, A_{5}$ (a.u.) of a crystalline dilute $C u_{99 \%} M n_{1 \%}$ alloy (from [20]) and of an icosahedral AlMnSi QC (from [22]). On this figure, only the $T$-dependence is significant: The $C, A_{3}$ and $A_{5}$ data have been arbitrarily vertically shifted to be presented altogether. The lines are fits used to deduce the critical exponents.

decreased only by a small factor, exhibiting the same qualitative behavior as in the periodic dilute alloy $C u M n$ (Fig. 2). Quantitatively, they could be accurately analyzed in terms of power laws of $T /\left(T-T_{c}\right)$ (The $T_{c}$, here $5.42 \mathrm{~K}$, is slightly smaller than $T_{f}$ like in $C u M n$ ). The critical exponents were found identical to those for $C u M n[20]$ and many other $3 \mathrm{D}$ spin glasses.

The second challenge was to determine the spin value $S$ of the magnetic moments and their concentration $x$. Of course, this cannot be done from the only measurement of the term linear in field of $M(H)$ which yields the Curie constant and thus only the value of the product $x S(S+1)$. One can expect to get an independent determination of $x$ and $S$ from the analysis of the $M(H)$ curvature. But the analysis of $M(H)$ is not simple. It is because the magnitude of the magnetic interactions energy scale compare with the value of $\mu_{B} H\left(\mu_{B} H / k_{B}=5.4 \mathrm{~K}\right.$ for $H=8$ Tesla available in standard magnetometers). These interactions are the RKKY ones responsible for the spin glass transition $\left(T_{f} \approx\right.$ a few Kelvin) and the Kondo one (energy scale $T_{K} \approx 3.3 \mathrm{~K}$ in $A l P d M n$ QCs: See Section 4$)$. However, in the non linear susceptibility study close to the transition, a careful quantitative comparison of the $A_{3} / C$ ratio ${ }^{1}$ in $A l M n S i$ QC and $C u M n$ (where the spin value is known) could provide the value of the spin value in the $\mathrm{QC}: S \approx 3.7$ [22]. This rather large value of $S$ (> $>5 / 2$ expected at maximum) could originate in the presence of ferromagnetic pairs of $5 / 2$ $M n$ spins involved in the transition. From $S$ and the Curie constant, one found a moment concentration equal to $0.27 \%$ whereas the concentration of $M n$ is $21 \%$. Other works, taking into account the RKKY interactions effects in the analysis of $M(H)$, concluded also a rather large value of the $M n$-spin, about $5 / 2[17,19,23]$. We note that all these determinations of $S$ were carried when assuming implicitly a bimodal distribution of the spin values: either 0 or a finite value $S \neq 0$.

\subsection{More on the fraction of magnetic Mn atoms}

From the previous estimate of the $M n$ spin value and the measured Curie constants, one deduces that, at maximum, a few $\%$ of the $M n$ atoms are magnetic. Besides, NMR studies, the very early one of Warren et al. on metastable AlMn QCs [3] and those subsequently performed on stable AlPdMn QCs [24,25] conclude that most $M n$ atoms do not carry a magnetic moment $(S=0)$. A precise determination of the proportion of non magnetic $M n$ atoms cannot be obtained because of the uncertainty on line intensity

\footnotetext{
${ }^{1} C, A_{3}, A_{5}$ are all proportional to $N x$ but their dependence on $S$ is different. So the ratio $A_{3} / C$ depends on $S$ but not on $x$. Using classic magnetic moments $\mu$ instead of quantum spins $S$, one has: $C=N x \mu^{2} / 3 k_{B}, A_{3}=N x a_{3}(T) \mu^{4} / k_{B}^{3}$ and thus $A_{3} / C \propto \mu^{2}$. For a paramagnet with no interactions, $a_{3}$ is constant, equal to $1 / 45$ as given by the series expansion of the Langevin function, i.e. the classic limit for the Brillouin function. For 3D spin glasses, $a_{3}(T)=b_{3}\left[T /\left(T-T_{c}\right)\right]^{\gamma}$ where $b_{3}$ is of constant value, found experimentally to be close to $1 / 45$ in standard $M n$-based spin glasses [20,22]. Thus $A_{3} / C \approx\left[T /\left(T-T_{c}\right)\right]^{\gamma} \mu^{2} / 15 k_{B}^{2}$, which allows to deduce the value of $\mu$ once those of the transition temperature $T_{c}$ and the critical exponent $\gamma$ have been extracted from the temperature dependence of $A_{3}(T)$.
} 
measurements in NMR experiments. So, one can imagine the existence of other $M n$ atoms which would be magnetic (hence not contributing to the NMR ${ }^{55} M n$ signal) and would not contribute to the measured Curie constant. This should be the case of two $M n$ moments interacting via a strong AF interaction or more complex arrangements of correlated $M n$ spins, provided that their resulting spin is zero. The method which has to be used for detecting such spins depends on their fluctuation time. If the latter is long, these spins can be detected in specific heat experiments since they give rise to a magnetic hyperfine contribution $C_{\text {hyp }}=C_{N} / T^{2}$ to the specific heat. Indeed, $C_{N}$ takes into account all the localized moments (concentration $\left.x^{*}\right): C_{N} \propto N x^{*} S^{2}$, whatever their magnetic interactions and relative orientations, provided that the spins are frozen at the time scale (a few seconds) of the experiment. (Because of its $T^{-2}$ dependence, $C_{\text {hyp }}$ is only measurable at very low temperature, practically below $300 \mathrm{mK}$, and thus includes the contribution of the spins frozen in the spin glass interactions when $T_{f} \gg 300 \mathrm{mK}$.) The value of the product $x^{*} S^{2}$ deduced in this way has been systematically found to be very small and, so, could be measurable only for the more magnetic $A l M n$ and $A l P d M n$ QC samples $[4,16,17]$. It is precisely the case where $T_{f} \gg$ $300 \mathrm{mK}$ and where almost all the spins contributing to the Curie constant are involved in the spin glass phase (almost no Kondo spins: See section 4). One found a value of $x^{*} S^{2}$ well comparing with the Curie constant measured on the same QC samples. This discards the hypothesis of additional frozen AF pairs or clusters. Hence if strongly correlated groups of spin do exist, they must fluctuate (as a whole) at any temperature including below the spin glass transition, which implies that they must be entirely decoupled from the spins contributing to the Curie constant and the spin glass transition. Their existence remains a quite open question. ${ }^{2}$

In summary, a very few Mn atoms appear to carry a magnetic moment in AlMn and AlPdMn QCs. In the next section, the origin of these moments will be discussed as well as the parameters governing the moment formation.

\subsection{About the moment formation}

The first question is to know the location of the very few magnetic $M n$ atoms. First, they do not cluster in fine grains, as shown from the existence of a spin glass transition. Secondly, they do belong to the QC structure as proved from their effect on several bulk properties. Indeed, i) the anomalous Hall effect is directly related to the $M n$ magnetization in decagonal T-AlMn phases [4], ii) the electrical conductivity of AlPdMn single grains increases linearly with the concentration of magnetic $M n$ atoms (See ref. [26] and below Section 3) and iii) all ${ }^{27} \mathrm{Al}$ nuclear spins are sensitive to the conduction electron spin polarization induced by the localized $M n$ moments, as shown by several NMR studies [24,25]. Consequently, the challenge was to understand the conditions for moment formation in the QC structure.

In icosahedral AlMnSi and decagonal T-AlMn phases [4,19,22], as well as in the first synthesized AlPdMn single grains (containing about 9 at\% $M n$ ) [16,17], the concentration of magnetic $M n$ atoms, although sample dependent, was always close to $1 \%$. This could suggest the existence of specific $M n$ sites in the QC structure with environments favouring moment formation. Later on, the possibility to grow AlPdMn single grains containing slightly smaller $M n$ amounts (about 8 at\% $M n$ ) gave the opportunity to observe that the moment concentration could range actually over two decades, from $10^{-4}$ to about $1 \%$ for the studied samples [5,26]. Then a systematic study of AlPdMn QCs of various composition, focusing in particular on 14 different QC pieces cut in Czochralski grown single grains, could be performed [5,27]. Note that for such studies, it is important to avoid grain boundaries which could favor spurious $M n$ moment formation, thus clouding the issue when the moment concentration is very low. Although each QC piece had been previously annealed at $800^{\circ} \mathrm{C}$ in order to improve the structural homogeneity, the moment concentration was found to depend (within a factor about 2) on subsequent thermal treatments [5,27]. More precisely, the cooling rate after a heating at $800^{\circ} \mathrm{C}$ was found to be a pertinent parameter: The

\footnotetext{
${ }^{2}$ Inelastic neutron scattering should be a quite suitable tool in order to detect such correlated spins through the measurement of time and space spin correlations. One of the present author (JJP) has carried such experiments in collaboration with L.P. Regnault (CEA Grenoble) on spectrometers IN 22 and IN 8 at Institut Laue Langevin (ILL-Grenoble). No magnetic signal could be detected up to now in the $\mathrm{Q}$ range where it should appear if one assumes that the correlated $M n$ spins are located on vertices of $M n$ icosahedra with identical orientation, in agreement with structural models (See Section 2.3).
} 

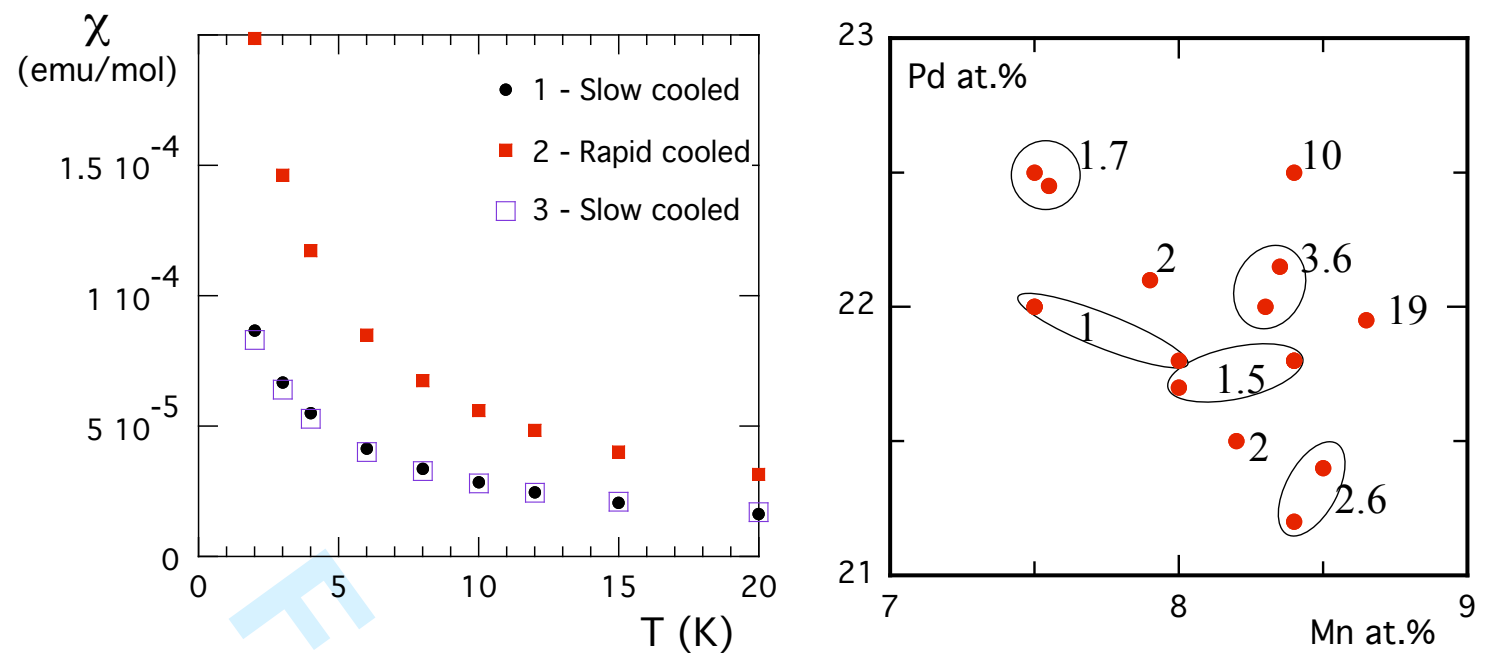

Figure 3. Left : Influence of the thermal treatment on the magnetic susceptibility of a i- $A l_{69.8} P d_{21.8} A l_{8.4} \mathrm{QC}$ single grain. Starting from state 1 , obtained from a slowly cooling from $800^{\circ} \mathrm{C}$ at a rate of $10^{\circ} \mathrm{C} /$ hour, the concentration of magnetic $M n$ atoms increases by a factor 2 after a heating at $800^{\circ} \mathrm{C}$ followed by a rapid cooling at a rate of $150^{\circ} \mathrm{C} / \mathrm{mn}$ (state 2 ). A new heating at $800{ }^{\circ} \mathrm{C}$ followed by a slow cooling at a rate of $10^{\circ} \mathrm{C} /$ hour (state 3) allows to recover the same moment concentration as in the initial state 1.

Right: Composition dependence of the magnetic $M n$ concentration (deduced from the Curie constant assuming $S=5 / 2$ ): Relative values are given, 1 being assigned to the less magnetic sample studied. The $P d$ and $M n$ concentration are measured by XWDS and ICP-OES. All the samples are in the same annealing state: Cooled from $800^{\circ} \mathrm{C}$ at a cooling rate of $10^{\circ} \mathrm{C} /$ hour.

slower the cooling rate, the smaller the moment concentration (Fig.3). Besides, for a given cooling process, the moment concentration depends strongly on the alloy composition: See Fig. 3. By contrast the exact structural state (the so called $F, F_{2}, F_{2 M}$ phases) was found to have no influence [5].

One observes that the concentration of magnetic $M n$ atoms is minimum for a composition about $7.75 \%$ of $M n, 21.9 \%$ of $P d$ and increases drastically when the $M n$ as well as the $P d$ amount changes (Fig.3). These results allowed to clear up the origin of the large scattering of the magnetization amplitudes of $A l P d M n$ samples reported in the literature [5]. They suggest that magnetic $M n$ in $A l P d M n$ QCs are not located on particular sites but instead most probably associated with defective local arrangements of the various chemical species in the neighbouring of the $M n$ atom which becomes magnetic. One can conjecture the existence of an ideal composition for which no localized moments would be present, provided that the structure is entirely relaxed (See below).

This conclusion is different from that reached from the study of crystalline AlMn and AlPdMn phases. In the hexagonal $\mu A l_{4} M n$ phase, most of the $M n$ atoms are non magnetic but experiments and theoretical calculations both suggest the presence of magnetic moment on a given $M n$ crystallographic site [28, 29]. The same conclusion has been reached from the study of the 1/1-AlPdMnSi approximant [30]. In these two cases, moment formation occurs on particular sites, intrinsic to the structure. Note that these two crystalline phases, as well as the $M n$-rich T-AlPdMn, are exceptions. No $M n$ atoms are magnetic in most of the crystalline $A l M n$ and $A l P d M n$ phases, for instance in $A l_{6} M n, A l_{12} M n, \beta-A l_{9} M n_{3} S i$, the 1/1 approximant $\alpha-A l_{73} S i_{10} M n_{17}$, the $M n$ poor T-AlPdMn phase or the $\xi^{\prime}-A l P d M n$ phase $[18,30]$.

So, the question is: Why are most $M n$ atoms non magnetic ? Answering this question requires to combine an accurate description of the quasicrystalline structure and criteria for moment formation. All spin polarized band structure calculations [30,31] show that the presence of a pseudo-gap in the local MnDOS impedes the formation of a local moment $(S=0)$. This is indeed the case of many $M n$ sites in QCs and approximants. Another result of these calculations is that magnetic moments exist on a small fraction of the Mn sites in QCs [31]. But, of course, the validity of this prediction strongly depends on that of the structural model used. In contrast, experiments and ab initio calculations show that most Mn atoms carry a moment in AlMn and AlPdMn liquids [32,33]. It could be tempting to try to establish a correlation between the local environment of a given $M n$ atom (number, nature and distances of its first neighbours) and its magnetic state. However this approach is too incomplete. $M n-M n$ interactions, mediated by the conduction electrons over large distances ( $5 \AA$ and more), have to be taken into account $[29,34]$. The interaction energy $\Phi_{M n-M n}$ between two $M n$ atoms depends on the magnetic moments $m_{1}$ and $m_{2}$ of the 

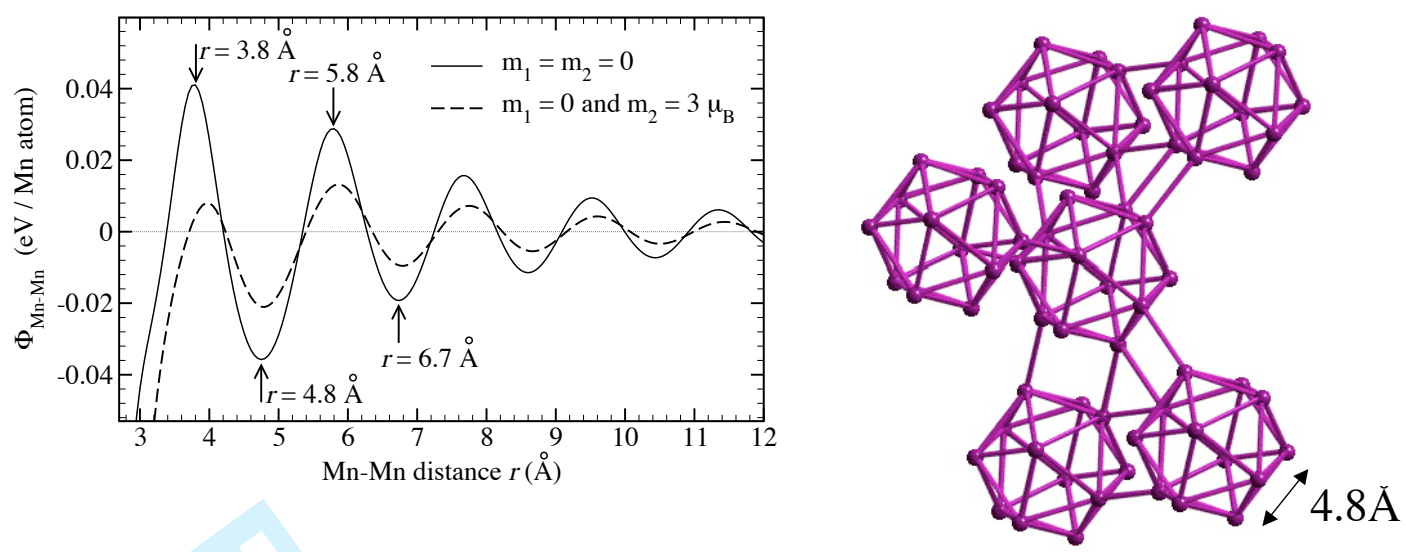

Figure 4. Left: $M n-M n$ interaction of two $M n$ atoms as a function of their relative distance $r$. The interaction depends on the $M n$ moments $m_{1}$ and $m_{2}$ (By courtesy of G. Trambly de Laissardière).

Right: piece of the $M n$ network deduced in the model of Ref. [36] (By courtesy of M. Quiquandon and D. Gratias)

atoms and on their relative distance $r$. One can develop $\Phi_{M n-M n}$ up to the second order with respect to $m_{1}$ and $m_{2}$ as follows: $\Phi_{M n-M n}\left(r, m_{1}, m_{2}\right)=a(r)+(b(r) / 2)\left(m_{1}^{2}+m_{2}^{2}\right)+c(r) \mathbf{m}_{\mathbf{1}} \cdot \mathbf{m}_{\mathbf{2}}$. (The development is correct for moments smaller than $\left.3 \mu_{B}\right) . a(r), b(r)$ and $c(r)$ have a sign oscillating with $r$ and a magnitude slowly decreasing with $r$, so that their range extends up to about $10 \AA$. If both $m_{1}$ and $m_{2}$ are null, the only contribution to the energy is $a(r)$, the potential energy between two non magnetic $M n$ atoms (when they are not nearest neighbors) [35]. The $c(r)$ term corresponds to the usual RKKY interaction between moments. The existence of the $b(r)$ term has strong consequences on the moment formation. Even if one of the atom of the pair is non magnetic, the interaction energy depends on the existence or not of a moment on the other atom. Indeed one finds a deep minimum of $\Phi_{M n-M n}$ for $r=4.8 \AA$ when the two $M n$ atoms are not magnetic. But moment formation on only one of the atoms would increase this minimum energy by $200 \mathrm{~K}$ (Fig. 4) and hence is not favorable. The same result, but even more marked, is found if the two atoms are magnetic [29]. These results have to be related with those of structural models, for instance that one for i-AlPdMn (and i-AlCuFe) recently published [36]. In this model, constructed with no fitting parameters and exhibiting a minimum number of different chemical configurations for the main atomic aggregates, the $M n$ atoms are found to form a remarkable network of icosahedra, connected by bridges in form of squares (Fig. 4). Strikingly the only first neighbor distance for $M n$ atoms on this network is precisely equal to $4.8 \AA$, which could explain the absence of magnetism in AlPdMn QCs. Note that in Ref. [36], the stoichiometry of an ideal i-AlPdMn phase is proposed to be $A l_{70.36} P d_{21.35} M n_{8.29}$, not far from the composition with the minimum moment concentration (See above).

In summary, the theoretical works explain why Mn atoms are not magnetic in the QC structure, unless local deviations from the ideal structure, necessarily present for concentrations different from the ideal one, induce moment formation.

\section{$3 \quad$ Interplay between magnetism and electronic transport}

The transport behavior of the first discovered QCs, which were metastable, was comparable with that of very resistive periodic or amorphous alloys. The situation changed radically when stable QCs were discovered. In 1990, it was shown that melt spun $A l C u R u$ samples could exhibit a resistivity $\rho$ reaching unexpected values, from 5000 to $30000 \mu \Omega \mathrm{cm}$ at $4 \mathrm{~K}$ depending on the composition, thus 15 to 100 times larger than in the most resistive standard alloys [37]. Soon after, other stable QC phases were shown to exhibit similar behavior, for instance $A l C u F e$ [38] and AlPdMn [39]. For all these phases, the conductivity $\sigma$ was shown to be sample dependent and to increase with the temperature. But, strikingly, the $\sigma(T)$ curves for different samples in a given system appear to be all parallel with one another (above $100 \mathrm{~K}$ ) exactly as the $\rho(T)$ curves of simple metals with different amounts of impurities. Another puzzling feature was 

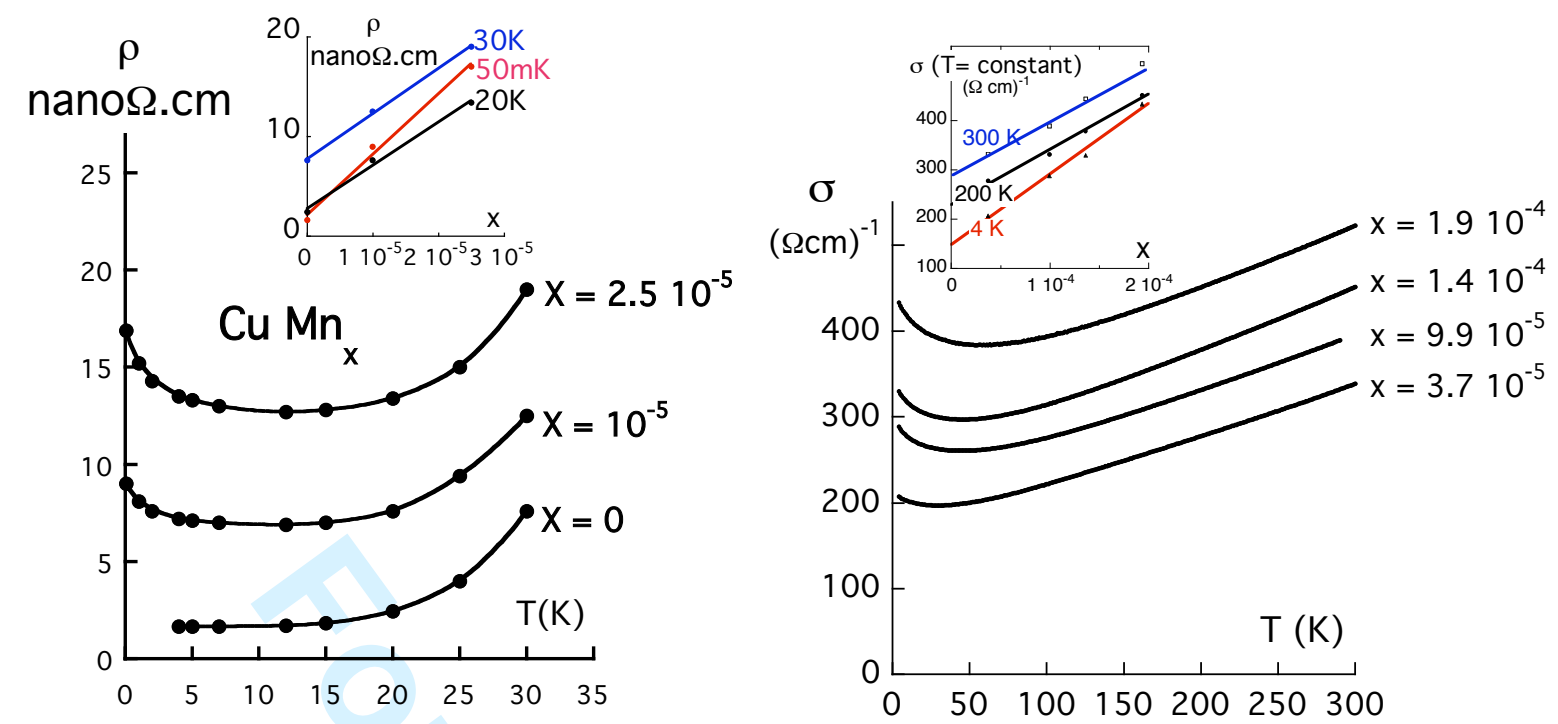

Figure 5. Left : Resistivity vs. $T$ for dilute $C u_{1-x} M n_{x}$ of different Mn concentration $x$ and vs. $x$ at different constant temperature (inset), from [40,41]. The curves are guides for the eye. Right: Same for AlPdMn QCs [26], except that $i$ ) $x$ denotes the actual moment concentration and not the total $M n$ concentration and $i i$ ) the ordinate is the conductivity $\sigma$ instead of the resistivity $\rho$.

a $\sigma$ decrease with improvement of the structural quality, in strong contrast with the simple metal case where the residual resistivity decreases with the defect concentration. A quite useful tool to understand the intrinsic character (i.e. in the absence of defects) of this very particular electronic diffusion is precisely the study of the disturbance of this diffusion caused by local defects in the quasiperiodic potential. Here, one faces the same challenge as for the resistivity in standard metals decades ago. Unfortunately, for a long time, such a quantitative study for QCs could not be carried out, due to the impossibility to find a way for detecting a very small concentration of local defects.

The understanding of the moment formation in the AlPdMn QCs gives a solution, at least in this phase. Indeed, a local defect in the quasiperiodic potential can be detected if it induces a moment on a neighboring $M n$ site. So, to obtain the concentration of such defects amounts to a measurement of the Curie constant which yields the moment (and thus defect) concentration. This hypothesis has been tested for a series of AlPdMn QC single grains by combining measurements of the conductivity and magnetization on the very same samples in the same annealed states [26]. A very simple relation between the conductivity $\sigma$ and $x$ has been found above $100 \mathrm{~K}: \sigma=\sigma_{0}(x)+\delta \sigma(T)$ where the residual conductivity $\sigma_{0}(x)$ increases linearly with $x$. As for other stable QC phases, $\delta \sigma(T)$ is sample independent. At lower temperatures, one observes an upturn of $\sigma(T)$, exhibiting a $\log (T)$ behavior and of amplitude also linear in $x$. So, this feature has been attributed to the Kondo effect (which results in a $\log (T)$-upturn of the resistivity at low temperature in simple metals). Thus, in QCs, the conductivity (instead of the resistivity) obeys a Mathiessen rule, i.e. is a sum of independent contributions:

$$
\sigma=\sigma_{0}(x)+\delta \sigma(T)+x K[\log (T)], \quad \sigma_{0}(x)=\sigma_{00}+a x
$$

This explains the striking similarity between $\sigma(T, x)$ of the QC and $\rho(T, x)$ of the very diluted $C u_{1-x} M n_{x}$, shown in Fig.5. Such a $\sigma$ law in QCs implies that the transport between two scattering events is not ballistic as shown in Ref. [42]. Interesting enough, this law appeared to apply only for samples exhibiting a vanishing concentration of defects, namely below $510^{-4}$, i.e. for defects distant by more than $30 \AA$ apart from one another. In view of the theoretical results for transport [43] and structural models (Section 2.3) for AlPdMn QCs, one can conjecture that the low value of the conductivity is due to an electronic confinment by the $M n$ icosahedra. Besides, the increase of $\sigma$ with the defect concentration could be related to the rapid decrease of this electronic localization in presence of a defect [43].

In conclusion, the thorough understanding of magnetism in AlPdMn QCs allowed to state quantitative 

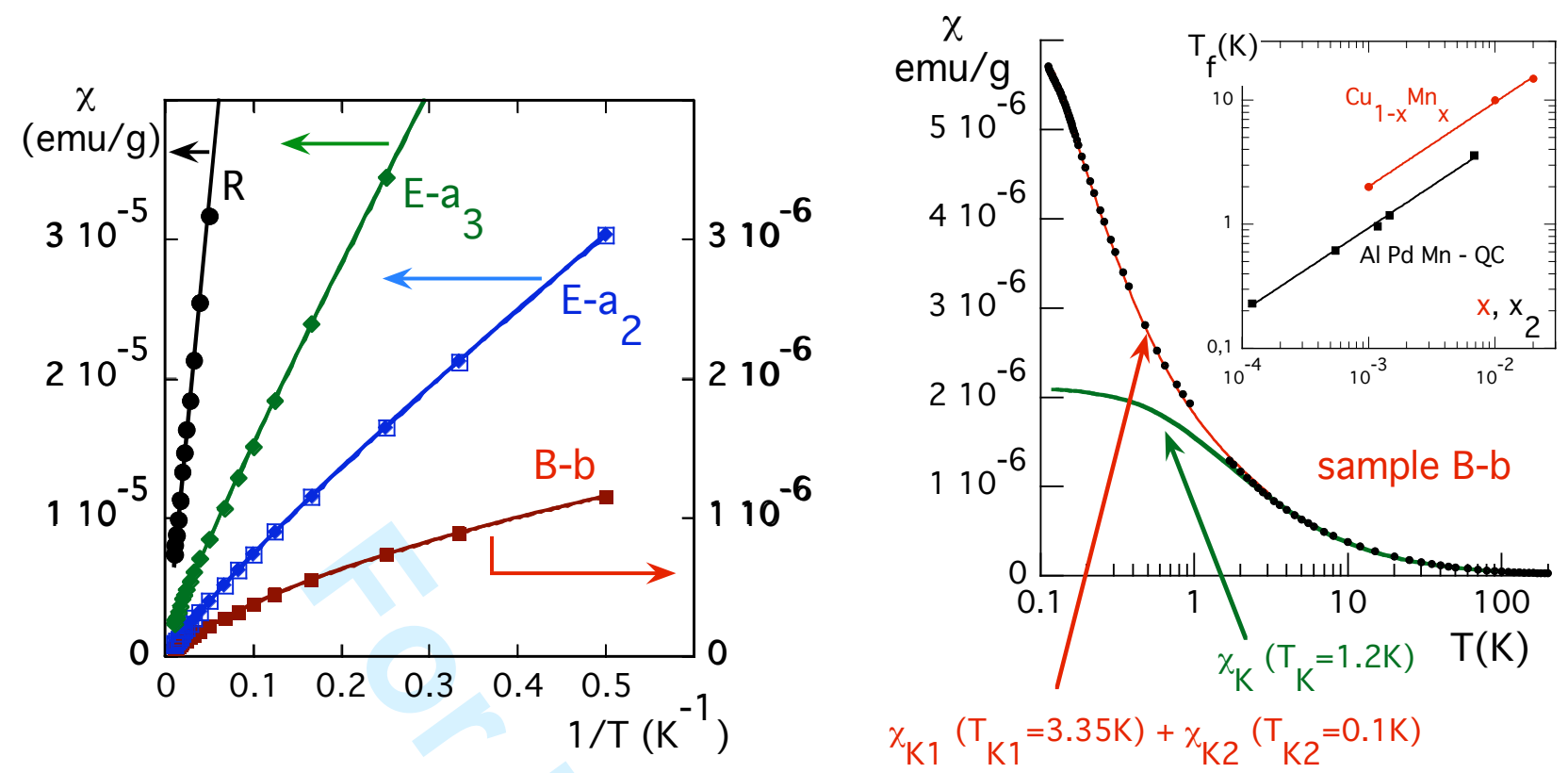

Figure 6. Left: The $\chi(1 / T)$ curvature for $A l P d M n$ QCs increases when the moment concentration decreases from that of sample $R$ down to that of sample $B-b$, found to be 100 times less magnetic than sample $R$ (For sample labels: See Ref. [5]). Right: $\chi(T)$ of sample $B-b$ in a semilog diagram and two fits using the susceptibility law for the $n=2 S$ Kondo model. A single $T_{K}$ law well accounts for the data from $2 \mathrm{~K}$ to $100 \mathrm{~K}$, vielding $T_{K}=1.2 \mathrm{~K}$, but is unable to account for additional data obtained over a third decade, here from 0.1 to $2 \mathrm{~K}$. In contrast the $\chi(T)$ can be well analyzed as the sum of two independent contributions over the whole temperature range: $\chi(T)=\chi_{K 1}\left(T, T_{K 1}\right)+\chi_{K 2}\left(T, T_{K 2}\right)$ with $T_{K 1}=3.35 \mathrm{~K}$ and $T_{K 2}=0.1 \mathrm{~K}$ [44]. Insert: Dependence of $T_{f}$ on $x_{2}$ compared to that on the moment concentration in the crystalline spin glass $C u M n$. The lines are power fits, yielding the same exponent value: 0.7 for AlPdMn QCs and CuMn alloys.

laws for the very unconventional conductivity behavior in QCs.

\section{Kondo vs RKKY interactions for the few magnetic $M n$}

Here, we enter upon a subject somewhat apart from the QC topic. As shown below, the AlPdMn QCs of very low moment concentration exhibit the characteristics necessary for a thorough study of the competition between the RKKY and the Kondo interactions. Because most of the standard dilute alloys do not present such interesting characteristics, it was worth undertaking this study [44], in order to bring information in a debated field in magnetism physics. Let us recall that the RKKY interaction $J_{i j}$ between moments $i$ and $j, r_{i j}$ apart, decreases with the distance as $1 / r_{i j}^{3}$. So, when the moment concentration is vanishing, most local moments undergo almost vanishing $J_{i j}$ couplings. Then, the Kondo interaction (energy scale: $T_{K}$ ), which is a single moment interaction with the conduction electron spins, may become dominant. For intermediate moment concentrations, the problem of the RKKY $\times$ Kondo competition becomes relevant. Theoretically, this problem remains a challenge in contrast with the two limiting cases (pure RKKY, pure Kondo) which are (now) well understood. The difficulty comes from the very same origin of the RKKY and Kondo interactions, i.e. the antiferromagnetic coupling between the spin of the local moment and the spins of the conduction electrons on the moment site.

The only mentioned magnetic interactions in the first discovered $A l$-based QCs with $M n$ were RKKY like, due to the magnitude of the moment concentration, about 1\% (Section 2.3). But once AlPdMn QC single grains of much lower moment concentration could be synthesized, the Kondo interactions could be detected. Let us recall several Kondo properties. The Kondo coupling results in the formation of a non magnetic singlet at low temperature, which induces a continuous negative curvature in the $\chi(1 / T)$ curve from the high temperatures down to $0 \mathrm{~K}$ where $\chi$ reaches a finite value $\chi(T=0) \neq 0$. In addition, in simple metals, the Kondo interaction is responsible for a characteristic $\log (T)$ upturn in the low temperature resistivity. The same features are observed in the less magnetic AlPdMn QCs, except that it is the conductivity which exhibits the $\log (T)$ upturn (Section 3). When plotting the susceptibility of AlPdMn 
QCs vs. $1 / T$ (Fig.6), one observes that $\chi$ is $1 / T$-linear (Curie behavior) above $T_{f}$ for the more magnetic sample denoted $R$ (Here, we use the notations of Ref. [5]). Hence the $R$ sample is a pure RKKY spin glass. But the other $\chi(1 / T)$ curves exhibit continuous curvatures which are more and more pronounced when the sample is less and less magnetic, thus when approaching the single moment limit, i.e. the pure Kondo case [44]. A Kondo fit of the $\chi(T)$ of the less magnetic sample $B-b$ performed in the $2 \mathrm{~K}-100 \mathrm{~K}$ temperature range provides a $T_{K}$ equal to $1.2 \mathrm{~K}$. The competition Kondo $\times$ RKKY manifests itself in two phenomena. First, the $\chi(1 / T)$ curvature attributed to the Kondo effect evolves progressively when one changes the moment concentration from that of sample $R$, with pure RKKY couplings down to that of sample $B-b$, with presumably dominating Kondo couplings (Fig.6). Second, for the very same sample, one observes the Kondo effect from the $\chi(1 / T)$ curvature at large temperature and the RKKY manifestation, i.e. a $\chi_{a c}(T)$ peak, at low temperature. So AlPdMn QCs meet with all the characteristics suitable for the Kondo $\times$ RKKY competition study, difficult to get in standard alloys because of the solubility limit (case of $\mathrm{Fe}$ in $\mathrm{Cu}$ ) and/or the too low $T_{K}$ value ( $5 \mathrm{mK}$ for $M n$ in $\mathrm{Cu}$ ). In the AlPdMn QC case, the $T_{K}$ lies in the Kelvin range and it is possible to get samples of moment concentration varying over two decades (Section 2.3). The recently published study performed on 22 samples [44] could answer to several questions concerning the competition effects. For instance, it was shown that the susceptibility does not reach the expected constant value at low temperature. This can be interpreted as the effect of the existence of two very different $T_{K}$ values: The larger one $\left(T_{K 1}\right)$ accounts for the high temperature $\chi(1 / T)$ curvature and the smaller one $\left(T_{K 2}\right)$ accounts for the lack of saturation of $\chi(T)$ at low temperature (Fig.6). More generally, when using very general Kondo physics arguments, and thus carrying out the data analysis with no preconceived model, a very robust and simple result was found: The magnetization $M(H, T)$ is a sum of two contributions. One can write:

$$
M(H, T)=\left[N g \mu_{B} S\right] x_{1} P_{1}\left(H, T, T_{K 1}=3.35 K\right)+\left[N g \mu_{B} S\right] x_{2} P_{2}\left(H, T, T_{K 2} \approx 0\right)
$$

where the $x_{i}$ s and the $P_{i}$ s have the dimension of a moment concentration and polarization respectively. The first contribution, of $T_{K 1}=3.35 \mathrm{~K}$, behaves as a pure Kondo magnetization, whereas the second contribution, of $T_{K 2} \approx 0$, appears to be purely RKKY like, directly responsible for the spin glass characteristics. In particular, it was shown that $T_{f}$ follows a power law of $x_{2}: T_{f} \propto x_{2}^{0.7}$ over the two available decades of $x_{2}$, exactly as it does for the archetypical pure spin glass $C u_{1-x} M n_{x}: T_{f} \propto x^{0.7}$, involving the same exponent value (Fig. 6). Here, the surprise comes from the domain of validity of Eq.3: Indeed, the later holds from the lowest moment concentrations (where $x_{1} \gg x_{2}$, i.e. with Kondo dominant) up to the concentration where the RKKY couplings dominate fully the magnetization (i.e. when $x_{2} \gg x_{1}$ ) and thus cannot be considered as a perturbation. For more details, one can refer to Ref. [44]. Note that, in view of the present Kondo analysis, the prefactor of the $\log (T)$ term in Eq. 2 for the conductivity should be $x_{1}$ instead of $x$. However, only the less magnetic samples exhibit the transport behavior illustrated in Fig. 5 and for these samples $x_{2} \ll x_{1}$, so that $x$ can be identified with $x_{1}$ [44].

In summary, the diversity of the temperature dependence of the magnetization in $A l$-based QCs containing $M n$ is fully understood. It results from the sample dependent relative weight of Kondo and RKKY interactions. Besides, the QC study allowed to enlighten on the effects of the Kondo $\times$ RKKY competition from the Kondo dominant regime to the RKKY dominant regime.

\section{$5 \quad \mathrm{R}-\mathrm{Mg}-\mathrm{Zn} \mathrm{QCs}$}

In the F-type $R M g Z n$ and P-type $R M g C d$ QCs, with $R=T b, D y, H o, G d$ in concentration around 9\%, a Curie-Weiss law and a spin glass like freezing are observed (see [13] and references therein). The measured Curie constants agree with those calculated assuming a free $R^{3+}$ effective moment. Any significant crystal field effect is therefore undetectable from the susceptibility data. In $R M g Z n$ QCs, a peak in the ac susceptibility at $T_{f}(5.8 \mathrm{~K}$ for $R=T b, 3.6 \mathrm{~K}$ for $R=D y)$ and magnetization irreversibilities below $T_{f}$ suggest a spin glass ordering [14], the $R$ moments being coupled via RKKY interactions. The case of the more recently discovered $R M g C d$ QCs is less clear : a broad transition is observed in single grains but 
several anomalies occur in polycrystalline samples $[13,15]$. Hence we will mainly focus in the following on the much more studied $R M g Z n$ QCs in which the existence of a spin glass transition is strongly supported by the observation of an anomaly in the third order susceptibility $\left(A_{3}\right.$ term with the notations of section 2.1) above $T_{f}$ and by the fact that $T_{f}$ is proportional to $x^{0.66}$ in $Y_{1-x} T b_{x} M g Z n$ and $Y_{1-x} G d_{x} M g Z n$ QCs, where the magnetic rare earths $\mathrm{Tb}$ or Gd are substituted by non magnetic $Y$ [14]. Such a law, with this exponent value, is common to all RKKY spin glasses and also observed in AlPdMn QCs (Fig.6). However, a striking feature is that the Curie-Weiss temperature $\Theta$ is $i$ ) positive (using the definition $\chi=C /(T+\Theta)$ ), indicating dominant AF interactions between the $R$ moments, ii) much larger than $T_{f}$. The ratio $\Theta / T_{f}$ is close to 4 in all studied cases $(R=T b, D y, H o$ and $E r)$. This feature is reminiscent of the situation encountered in the geometrically frustrated periodic systems [45]. The possibility of an unusual spin freezing in $T b M g Z n$ has indeed been suggested [46].

The most fascinating properties of $R M g Z n$ QCs were revealed from neutron diffraction experiments $[13,47]$. In $H o M g Z n$, a strong magnetic diffuse scattering, due to short-range spin correlations (extending over about $10 \AA$ ), is observed below $5 \mathrm{~K}$, i.e. definitively above $T_{f}=1.95 \mathrm{~K}$. The magnetic diffuse scattering occurs outside the intense nuclear Bragg peaks, indicating AF short-range order, and exhibits an isosahedral symmetry. It can be accounted for by assuming short range spin correlations in $6 \mathrm{D}$ with a magnetic vector modulation $(3 / 4,0,0,1 / 2,3 / 4,1 / 2)$. In the real space, most $H o$ moments are located on a network of edgesharing dodecahedra (with edge length equal to $5.5 \AA$ ). The analysis of the spin correlations, assuming classical spins, on a single dodecahedron leads to diffuse scattering patterns close to the observed ones, despite a simplified hypothesis on exchange bounds.

\section{Conclusion}

At the present time, one has an unified view of the $M n$ magnetism in $A l$-based QCs. The few moments are randomly distributed in the structure. One can assume that the moment formation originates in defects which locally break the ideal quasiperiodic potential, the same defects which affect drastically the electronic transport. In the case of QCs containing rare earth atoms $R$, there are still many unresolved issues. More information is needed on the dynamics of the correlations, although recent studies did tackle this point $[48,49]$. Determining the nature of the freezing probably requires a careful analysis of the critical behaviour above $T_{f}$, as done for $A l M n$ QCs (section 2.1). Finally an open and much debated question is why one has not yet observed long-range AF order in a real QC. From a theoretical point of view, no symmetrybased argument forbids the existence of a quasiperiodic long-range magnetic order [12]. Indeed theoretical models of magnetism on frustrated and non frustrated quasiperiodic tilings may exhibit long-range AF order [50-52]. However in these models, only first neighbor interactions are usually considered whereas, in all magnetic QCs known up to now, the interactions are of the RKKY type, i.e. long ranged and of variable sign. This may be an important point [52]. One can try to sketch the main points required for a fruitful comparison of models and experiments. Interactions beyond first neighbors have to be taken into account in models. Frustration obviously plays a central role both in models and in real QCs. It could interesting to compare QCs with the much studied periodic frustrated systems, such as the pyrochlore compounds which contain $R$ tetrahedra. In these systems, frustration induces a rich variety of ground states [45], including non ordered ones (some of them exhibiting short range spin correlations [53]). Another point is to determine the crystal field effects in real QCs containing rare earths in order to know the nature of the ground state above $T_{f}$. Note that frustration on a quasiperiodic lattice may induce non colinearity for three or two dimensional spins [51]. Hence it is important to know whether spins in QCs are Ising or Heisenberg like. Finally, when studying magnetic properties of any new QC system, it is necessary to know precisely its structure and especially whether the magnetic atoms form a quasiperiodic array. Knowledge of the structure will in addition allows to quantify the importance of the geometrical frustration. In the case of transition elements, an additional essential question is to know whether they all carry a moment. If not and if the magnetic atoms do not occupy well defined sites forming a quasiperiodic array, nothing more than a usual spin glass order is expected in presence of RKKY interactions, as it is indeed the case in $A l P d M n$ QCs. This question is open in the case of the recently discovered $Z n M n S c$ and $Z n F e S c$ QCs [9]. 
More generally avoiding any kind of disorder is a essential in order to have a chance to observe AF order in a real $\mathrm{QC}$.

\section{Acknowledgments}

We would like to thank M. Audier, C. Berger, Y. Calvayrac, J.C. Lasjaunias, E. Lhotel, V. Simonet, A. Sulpice and G. Trambly de Laissardière who brought major contributions to many of the works cited in the present paper. We are also grateful to S. Bonnevault, D. Gratias, D. Mayou and M. Quiquandon for many fruitful discussions.

\section{References}

[1] J.J. Hauser, H.S. Chen and J.V. Waszczak, Phys. Rev. B 33, 3577 (1986)

[2] D. Shechtman, I. Blech, D. Gratias and J.W. Cahn, Phys. Rev. Lett. 53, 1951 (1984)

[3] W.W. Warren, H.S. Chen and G.P. Espinosa, Phys. Rev. B 34, 4902 (1986)

[4] A. Gozlan, C. Berger, G. Fourcaudot, R. Omari, J.C. Lasjaunias and J.J. Préjean, Phys. Rev. B 44, 575 (1991)

[5] F. Hippert, M. Audier, J.J. Préjean, A. Sulpice, E. Lhotel, V. Simonet and Y. Calvayrac, Phys. Rev. B 68, 134402 (2003).

[6] C.A. Swenson, T.A. Lograsso, N.E. Anderson and A.R. Ross, Phys. Rev. B 70094201 (2004).

[7] D. Rau, J. L. Gavilano, Sh. Mushkolaj, C. Beeli, M.A. Chernikov and H.R. Ott, Phys. Rev. B 68 134204 (2003).

[8] M. A. Chernikov, C. Beeli, E. Felder, S. Buchi and H.R. Ott, Phys. Rev. B 68094202 (2003).

[9] S. Kashimoto, S. Motomura, S. Francoual, S. Matsuo and T. Ishimasa, Phil. Mag. B 86725 (2006)

[10] J. Dolinšek, S. Vrtnik, M. Klanjšek et al., Phys. Rev. B 76, 054201 (2007).

[11] K. Binder and A.P. Young, Rev. Modern Physics, 58801 (1986).

[12] R. Lifshitz, Phys. Rev. Lett 802717 (1998) ; Mater. Sci. Eng. 294-296 508 (2000).

[13] T. J. Sato, Acta Crystallogr. A 6139 (2005).

[14] I. R. Fisher, K. O. Cheon, A. F. Panchula, et al., Phys. Rev. B 59308 (1999).

[15] S. E. Sebastian, T. Huie, I. R. Fisher, K.W. Dennis and M.J. Kramer, Phil. Mag. 841029 (2004).

[16] M.A. Chernikov, A. Bernasconi, C. Beeli, A. Shilling and H.R. Ott, Phys. Rev. B 48, 3058 (1993)

[17] J.C. Lasjaunias, A. Sulpice, N. Keller, J.J. Préjean and M. de Boissieu, Phys. Rev. B 52, 886 (1995)

[18] C.A. Swenson, I.R Fisher, N.E. Anderson, P.C. Canfield and A. Migliori, Phys. Rev. B 65184206 (2002).

[19] R. Bellissent, F. Hippert, P. Monod and F. Vigneron, Phys. Rev. Lett. 36, 5540(1987)

[20] R. Omari, J.J. Préjean and J. Souletie, J. Phys. (Paris), 44, 1069 (1983)

[21] D. Fiorani, J.L. Tholence and J.L. Dormann, J. Phys. C 195495 (1986)

[22] C. Berger and J.J. Préjean, Phys. Rev. Lett. 64, 1769 (1990)

[23] K. Fukamichi, in Physical Properties of Quasicrystals, ed. by Z.M. Stadnik, Springer series in Solid state sciences, Vol. 126, (Springer, Berlin, 1999), pp. 295-326.

[24] J.L. Gavilano, D. Rau, Sh. Mushkolaj, H.R. Ott, J. Dolinšek and K. Urban, Phys. Rev. B 65, 214202 (2002).

[25] V. Simonet, F. Hippert, C. Berger and Y. Calvayrac, J. Non-Cryst Solids 334-335, 408 (2004).

[26] J.J. Préjean, C. Berger, A. Sulpice and Y. Calvayrac, Phys. Rev. B 65, 140203 (2002).

[27] F. Hippert, M. Audier, J.J. Préjean, A. Sulpice, V. Simonet and Y. Calvayrac, J. of Non-Crystalline Solids 334-335, 403 (2004).

[28] V. Simonet, F. Hippert, M. Audier and G. Trambly de Laissardière, Phys. Rev. B 58, R8865 (1998).

[29] G. Trambly de Laissardière and D. Mayou, Phys. Rev. Lett. 85, 3273 (2000).

[30] F. Hippert, V. Simonet, G. Trambly de Laissardière, M. Audier and Y. Calvayrac, J. Phys.: Condens. Matter 11, 10419 (1999).

[31] J. Hafner and M. Krajči, Phys. Rev. B 57, 2849 (1998) ; M. Krajči , and J. Hafner, Phys. Rev. B 58, 14110 (1998).

[32] V. Simonet, F. Hippert, H. Klein, M. Audier, R. Bellissent, H. Fischer, A. P. Murani and D. Boursier, Phys. Rev. B 58, 6273 (1998).

[33] N. Jakse and A. Pasturel, Phys. Rev. B 76, 024207 (2007).

[34] G. Trambly de Laissardière, D. Nguyen-Manh and D. Mayou, J. of Non-Crystalline Solids 334-335, 347 (2004).

[35] J. Zou and A.E. Carlsson, Phys. Rev. Lett. 70, 3748 (1993).

[36] M. Quiquandon and D. Gratias, Phys. Rev. B 74214205 (2006)

[37] B.D. Biggs, S.J. Poon and N.R. Munirathnam, Phys. Rev. Lett. 65, 2700 (1990)

[38] T. Klein, C. Berger, D. Mayou and F. Cyrot-Lackmann, Phys. Rev. Lett. 66, 2907 (1991)

[39] P. Lanco, T. Klein, C. Berger, F. Cyrot-Lackmann, G. Fourcaudot and A. Sulpice, Phys. Europhys.. Lett. 18, 227 (1992)

[40] O. Laborde and P. Radhakrishna, J. Phys. F 3, 1731 (1973)

[41] P. Haen, J. Souletie and J. Teixeira, J. Low temp. Phys. 23, 191 (1976)

[42] G. Trambly de Laissardière, J.P. Julien and D. Mayou, this issue and Phys. Rev. Lett. 97026601 (2006) .

[43] G. Trambly de Laissardière and D. Mayou, Phys. Rev. B 552890 (1997).

[44] J.J. Préjean, E. Lhotel, A. Sulpice and F. Hippert, Phys. Rev. B 73214205 (2006) and cond-mat/0606241.

[45] A. P. Ramirez, in Handbook on Magnetic Materials, Vol. 13 (Elsevier Science, Amsterdam, 2001), pp.423-520.

[46] J. Dolinšek, Z. Zagličić, T. J. Sato, J. Q. Guo and A. P. Tsai, J. Phys.: Condens. Matter 157981 (2003).

[47] T. J. Sato, H. Takahura, A. P. Tsai, K. Shibata, K. Ohoyama and K. H. Andersen, Phys. Rev. B 61476 (2000).

[48] T. J. Sato, H. Takahura, A. P. Tsai and K. Shibata, Phys. Rev. B 73054417 (2006).

[49] T. J. Sato and A. P. Tsai, Phil. Mag. 872939 (2007).

[50] S. Wessel, A. Jagannathan and S. Haas, Phys. Rev. Lett. 90177205 (2003).

[51] E.Y. Vedmedenko, U. Grimm and R.Wiesendanger1, Phys. Rev. Lett. 93076407 (2004).

[52] E.Y. Vedmedenko, U. Grimm and R.Wiesendanger1, Phil. Mag. 86733 (2006).

[53] J. S. Gardner, S. R. Dunsiger, B. D. Gaulin et al., Phys. Rev. Lett. 821012 (1999). 
$\chi$ (emu/mole) 
age 1 1 f 36 Philosophical Magazine \& Philosophical Magazine Letters

\section{(a.u.)}

$10^{6}$

$10^{4}$

12

13

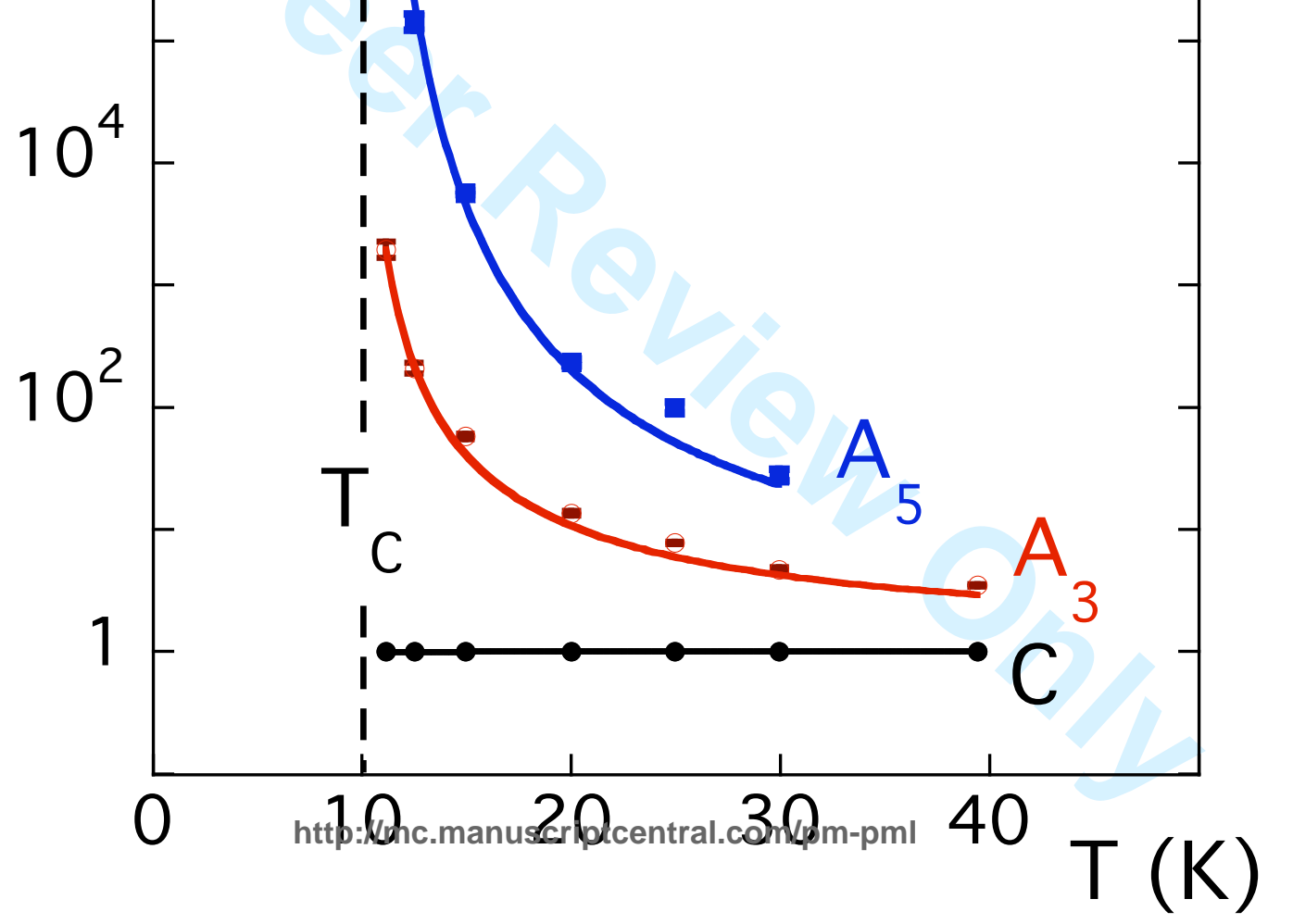

Crystalline $\mathrm{Cu}$ $+1 \% \mathrm{Mn}$ random

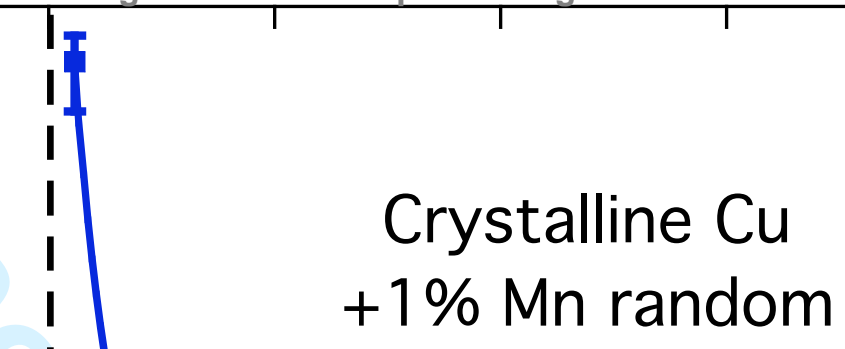


C, A Philosophical Magazine \& Philosophical Magazine Letters Page $\mathbf{1 6}$ of 36

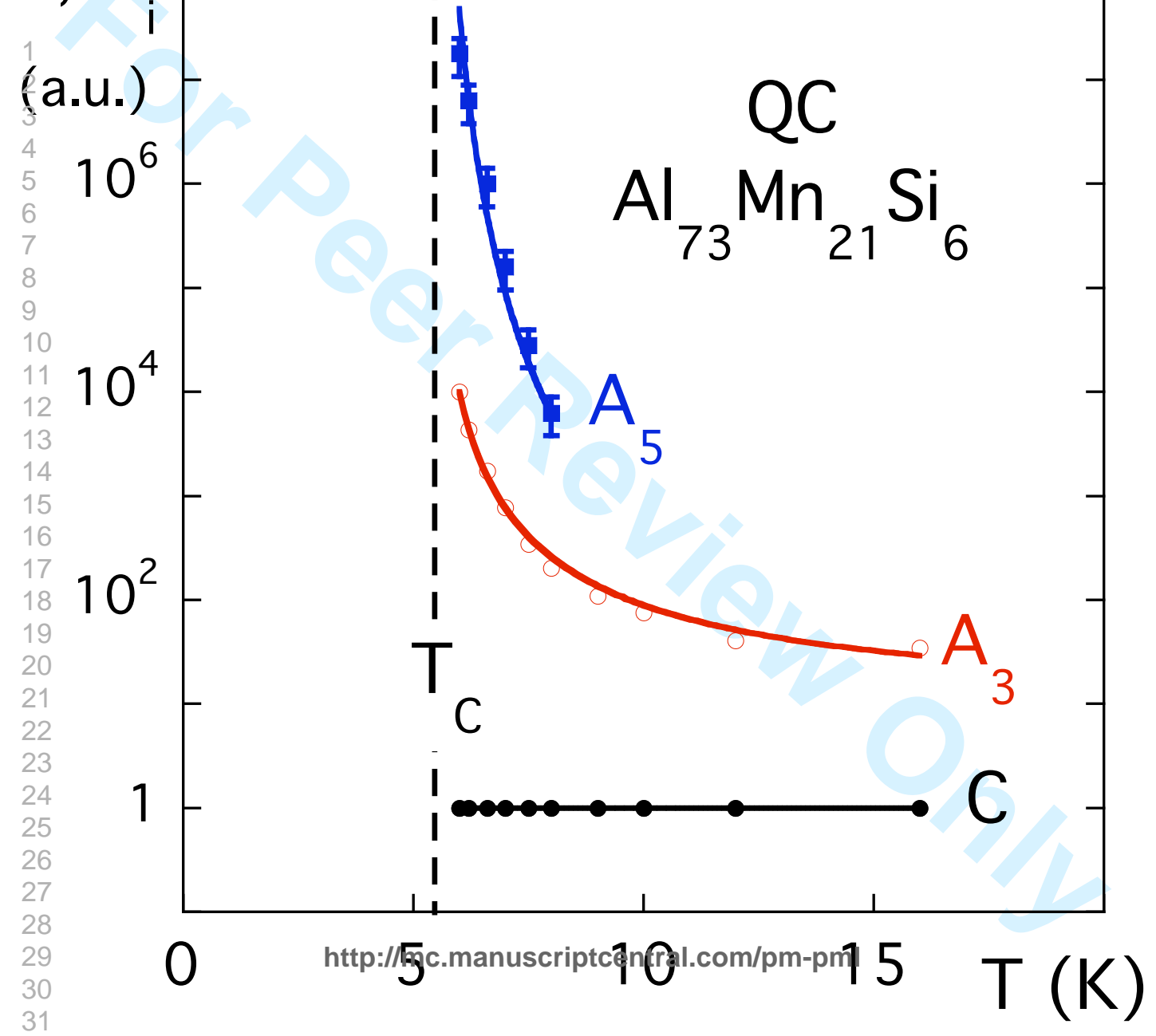




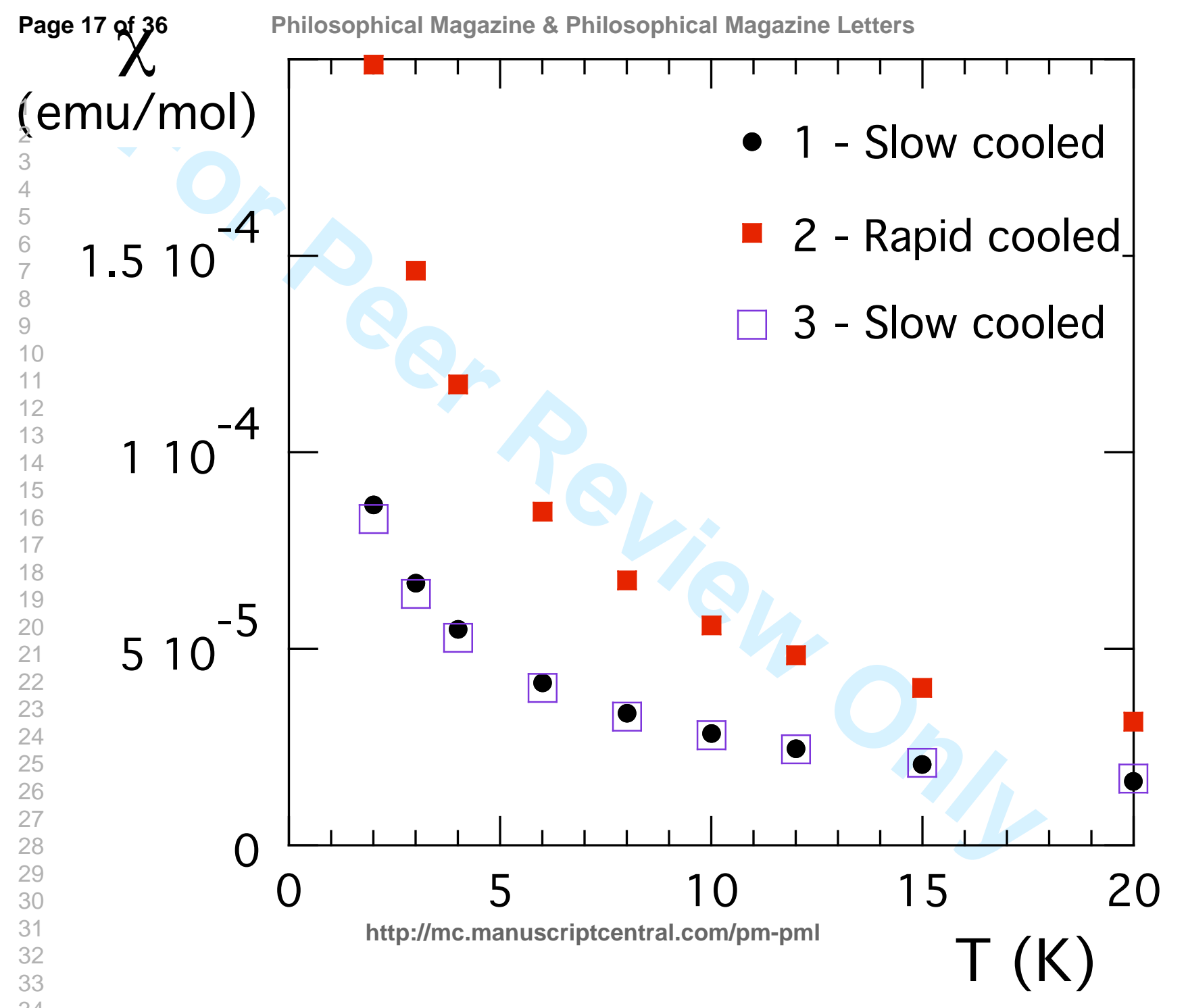


23 Philosophical_Magazine_\&_Phil|psophica_Magazine_LeRrgo 18 pf 36 $\mathrm{Pd}$ at.\%

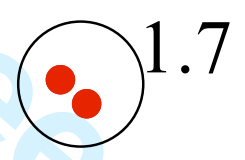

.10

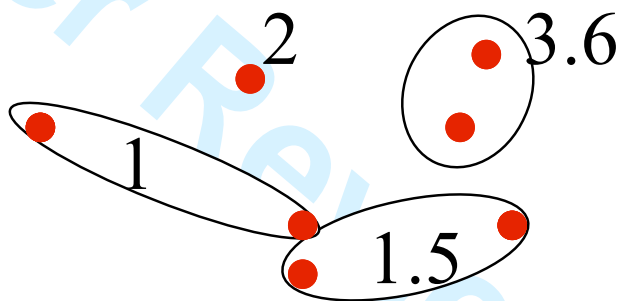

- 19

$\bullet 2$

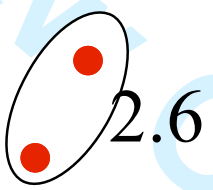

221

247

http://mc.manuscript\&entral.com/pm-pml

Mn at.\%

9 


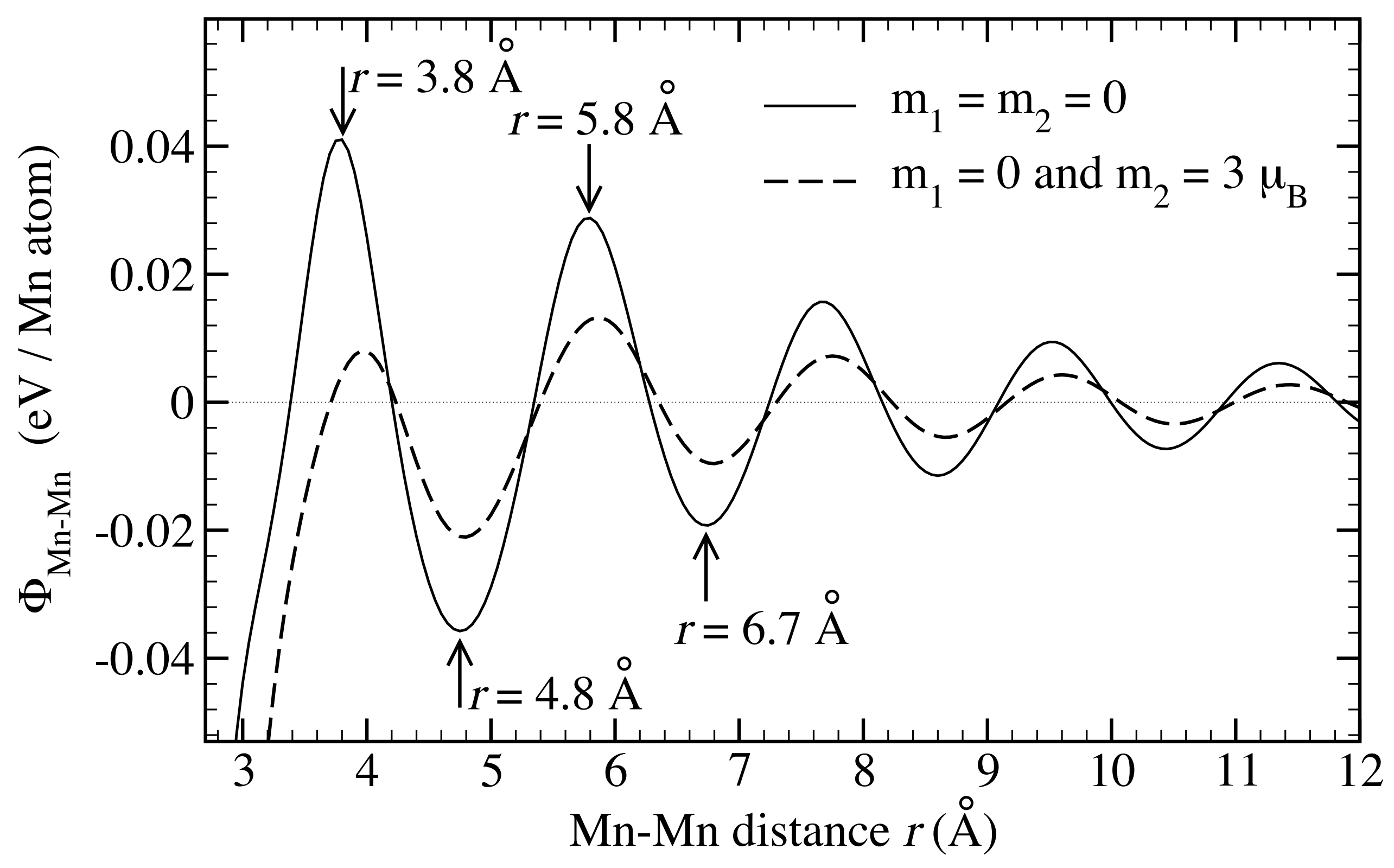

http://mc.manuscriptcentral.com/pm-pml 


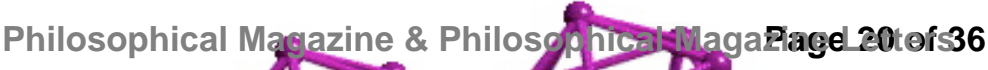

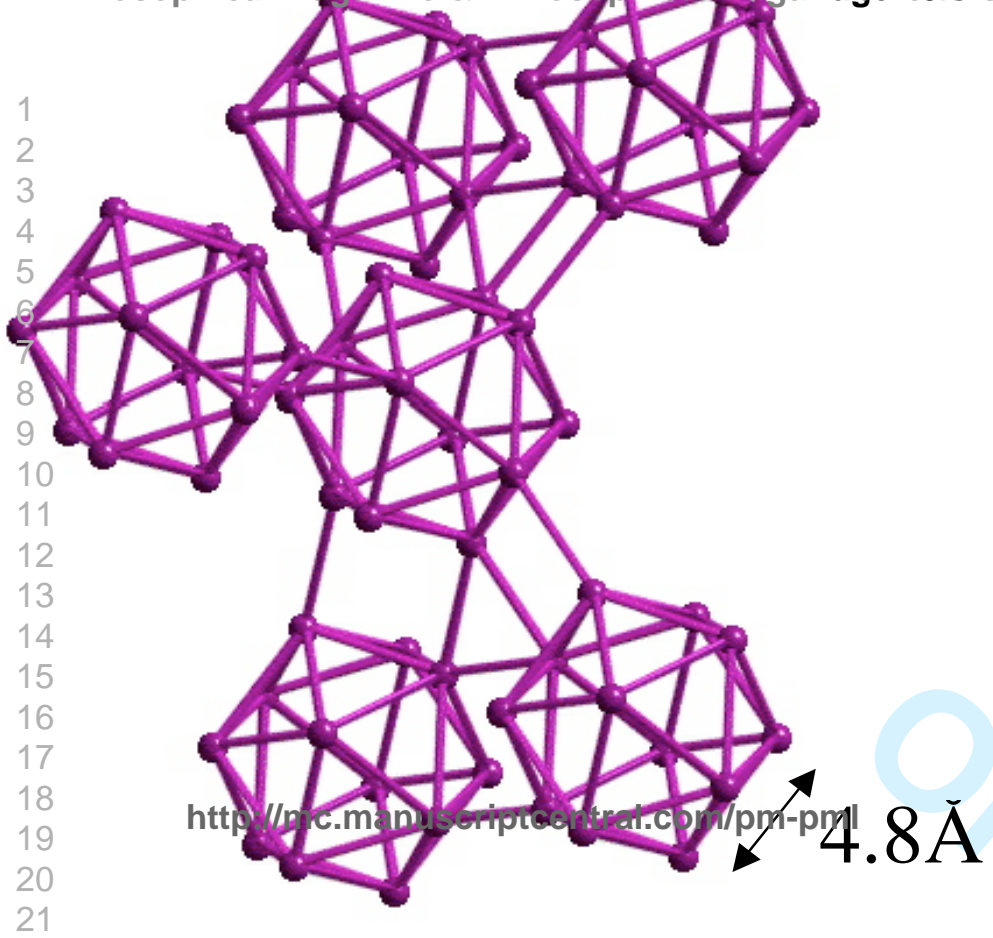




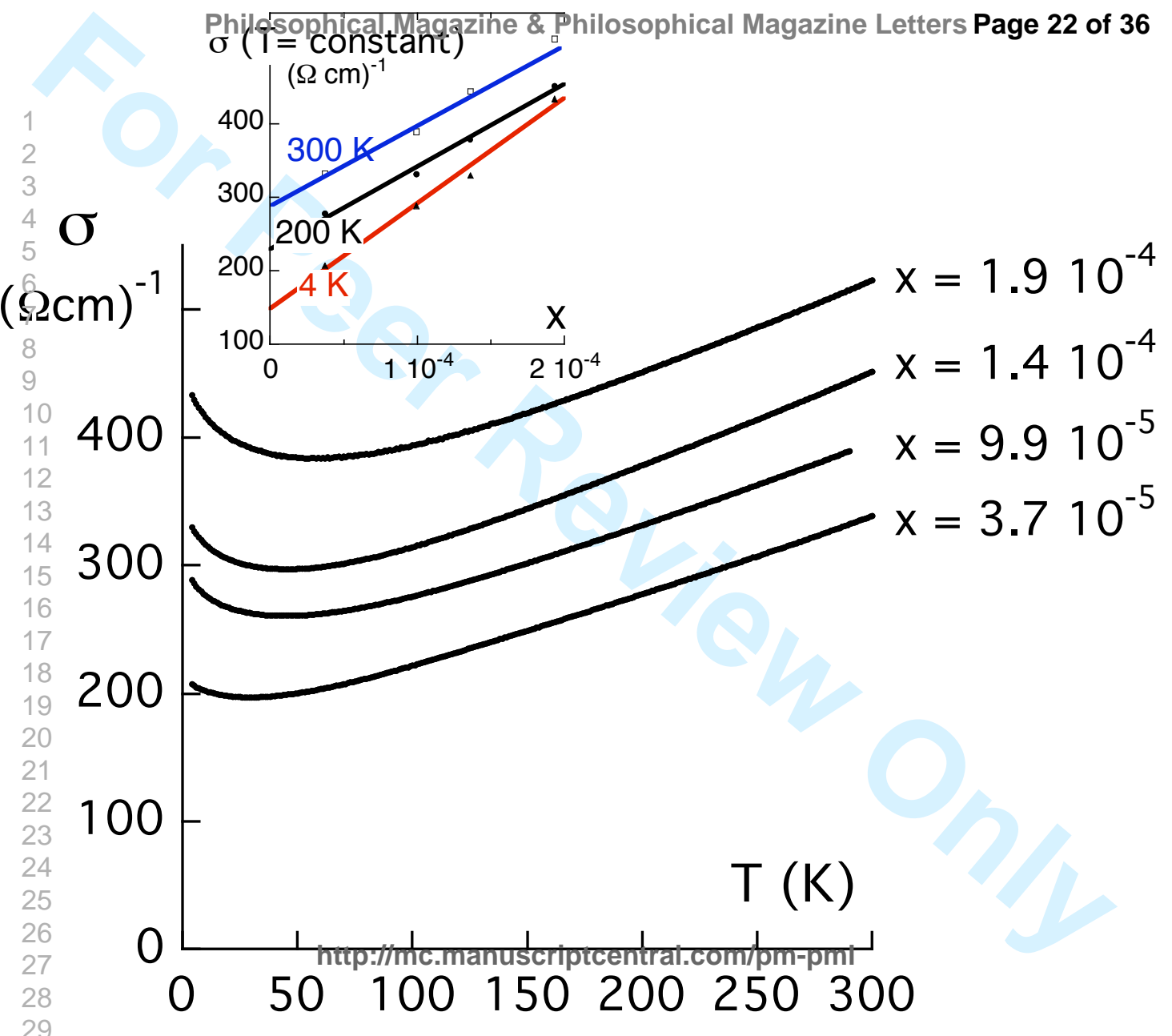


$\chi$ Philosophical Magazine \& Philosophical Magazine LetterBage 24 of 36

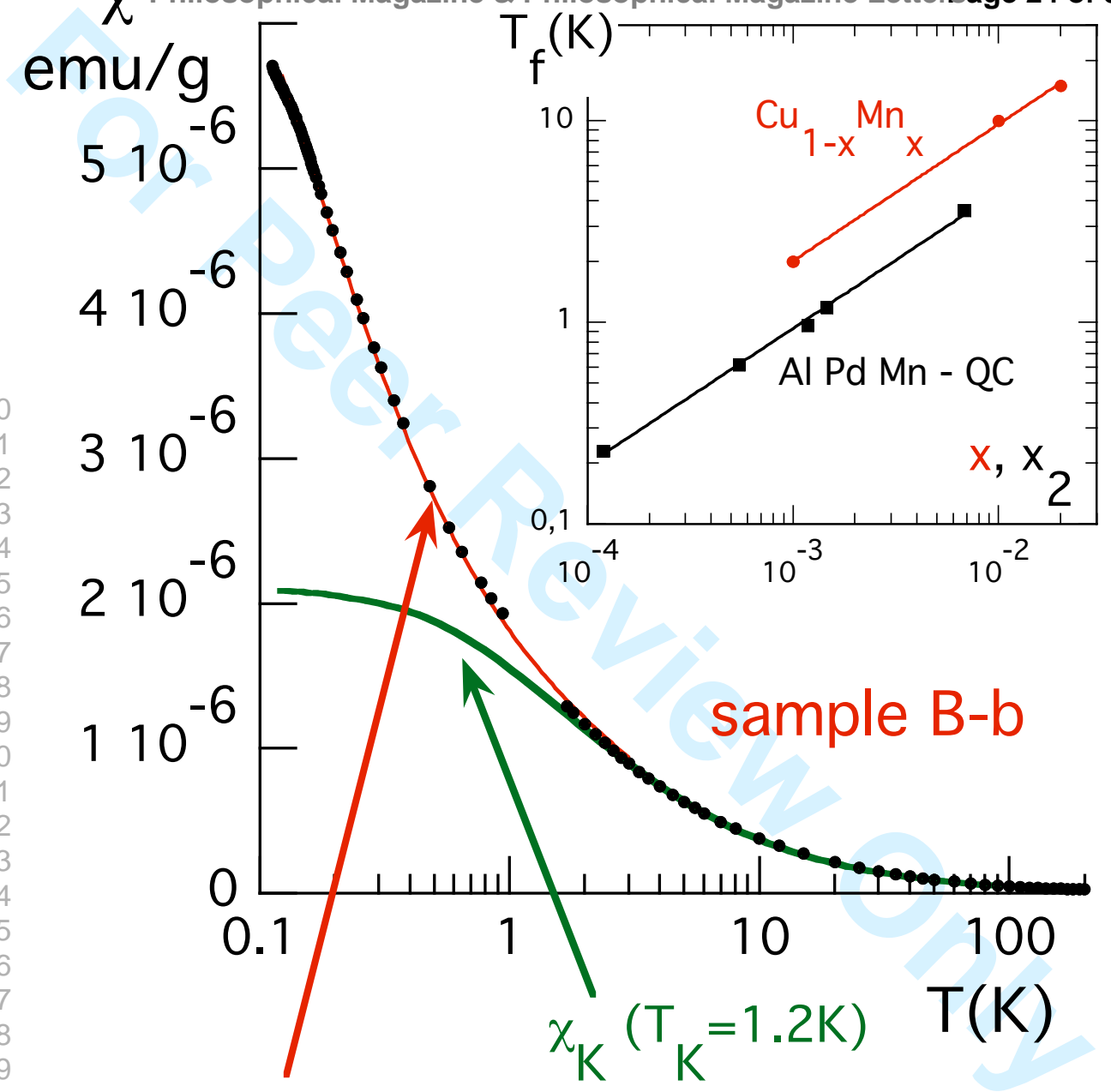

30

${ }_{32}^{31} \chi_{\mathrm{K} 1}\left(\mathrm{~T}_{\mathrm{K} 1}=3.35 \mathrm{~K}\right)^{\text {httpi/manuscriptcentral.com/pm-pml }}+\chi_{\mathrm{K} 2}\left(\mathrm{~T}_{\mathrm{K} 2}^{=0.1 \mathrm{~K})}\right.$ 


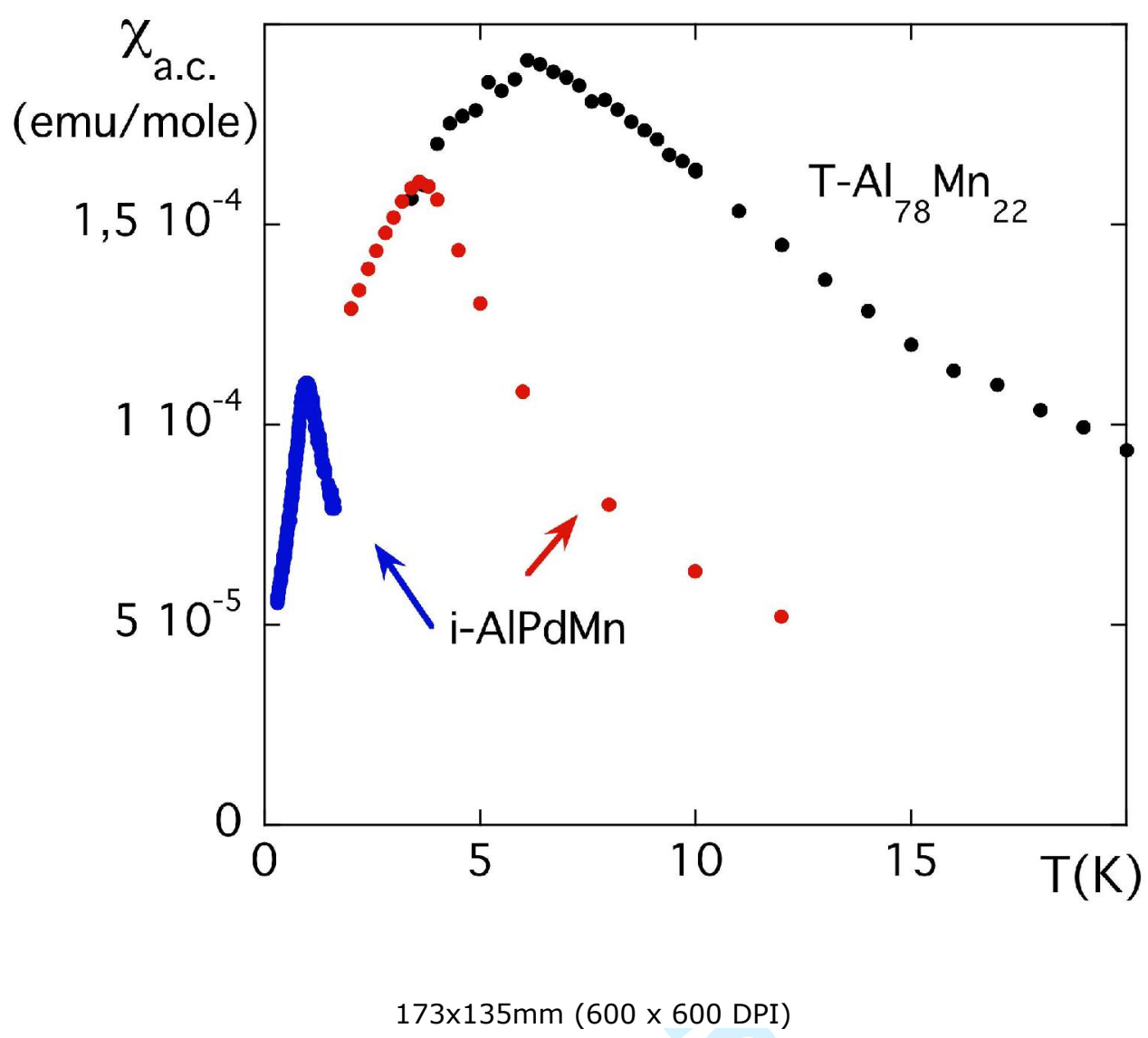

http://mc.manuscriptcentral.com/pm-pml 


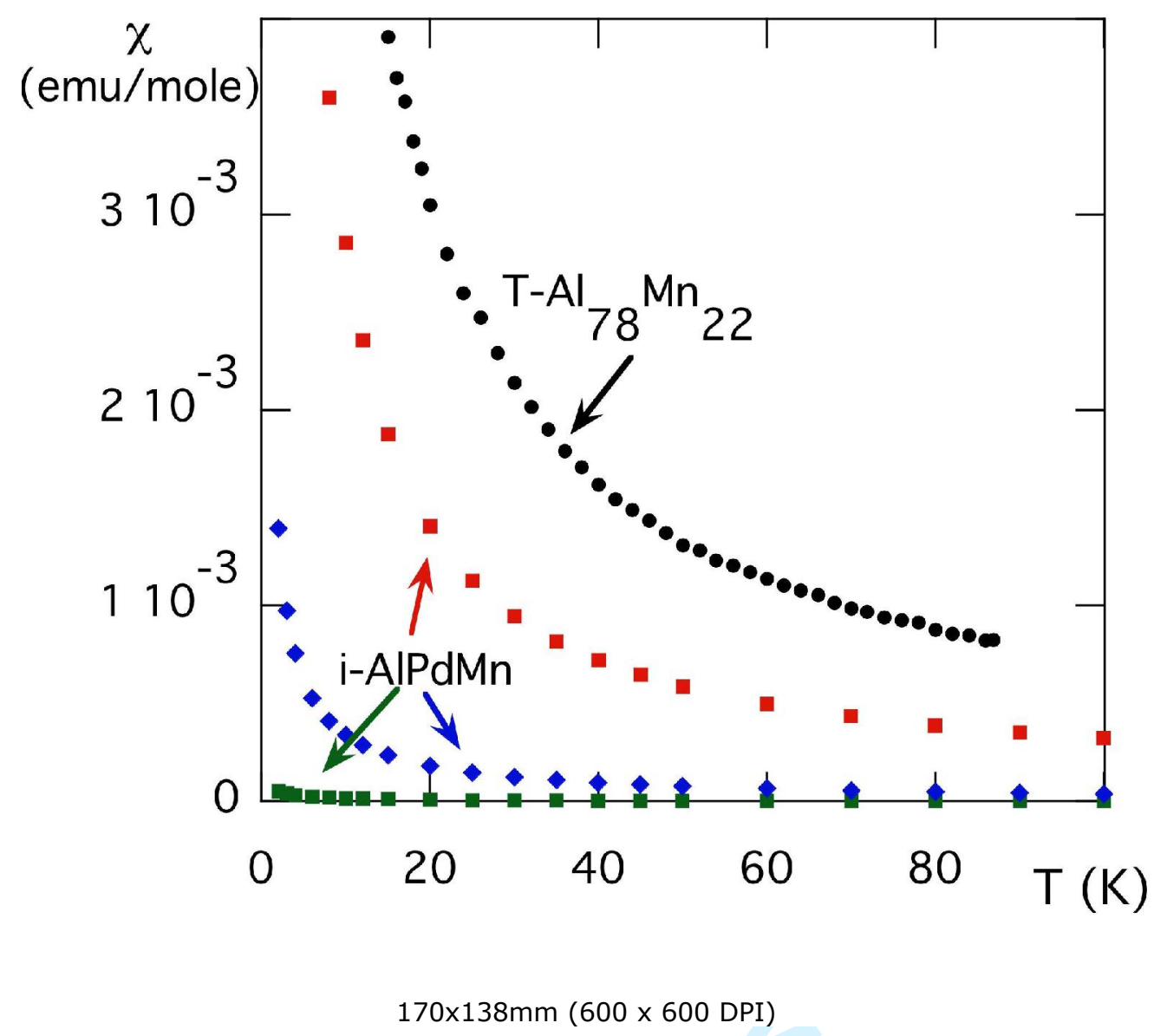

http://mc.manuscriptcentral.com/pm-pml 


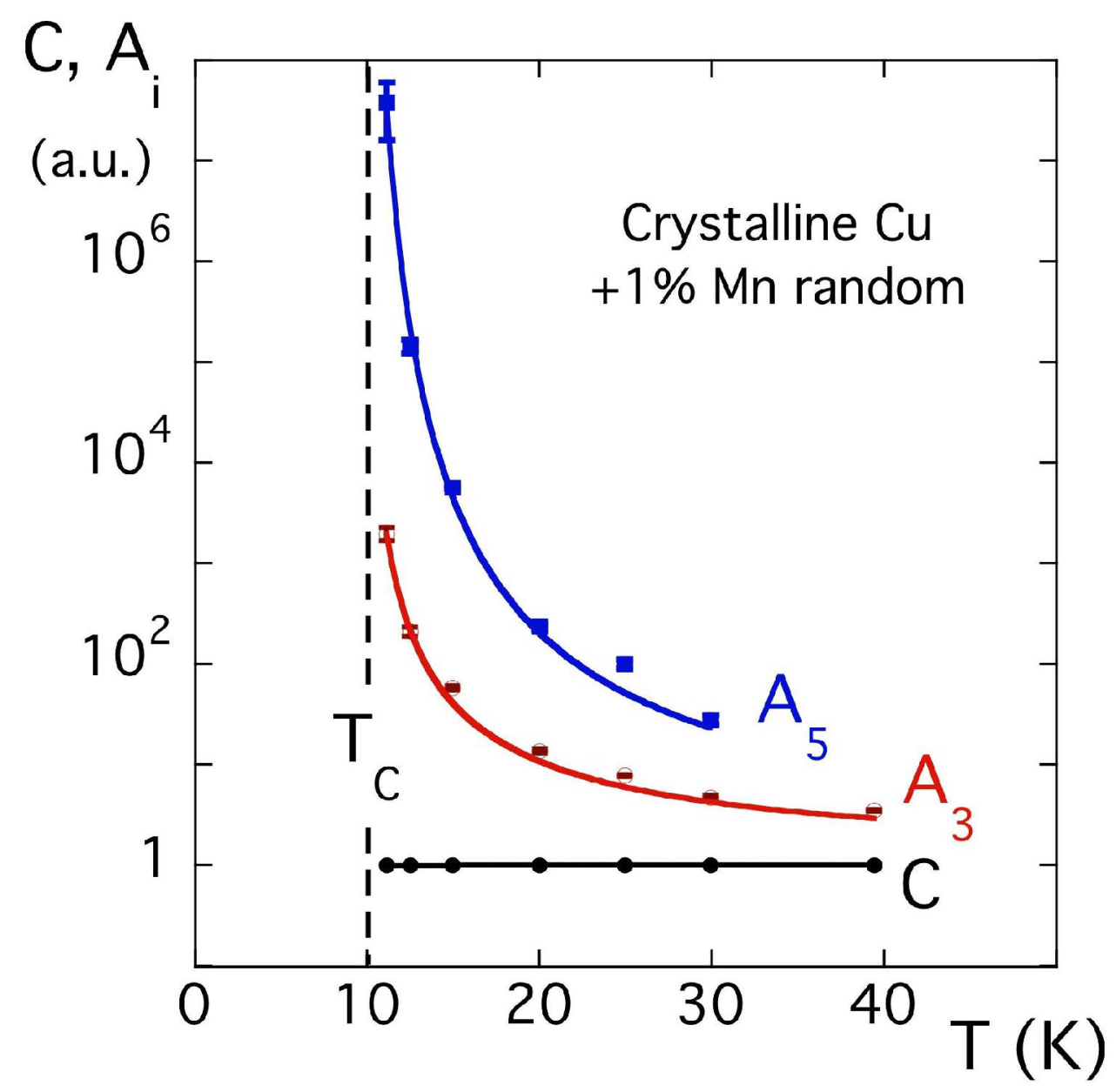

$150 \times 146 \mathrm{~mm}(600 \times 600 \mathrm{DPI})$ 


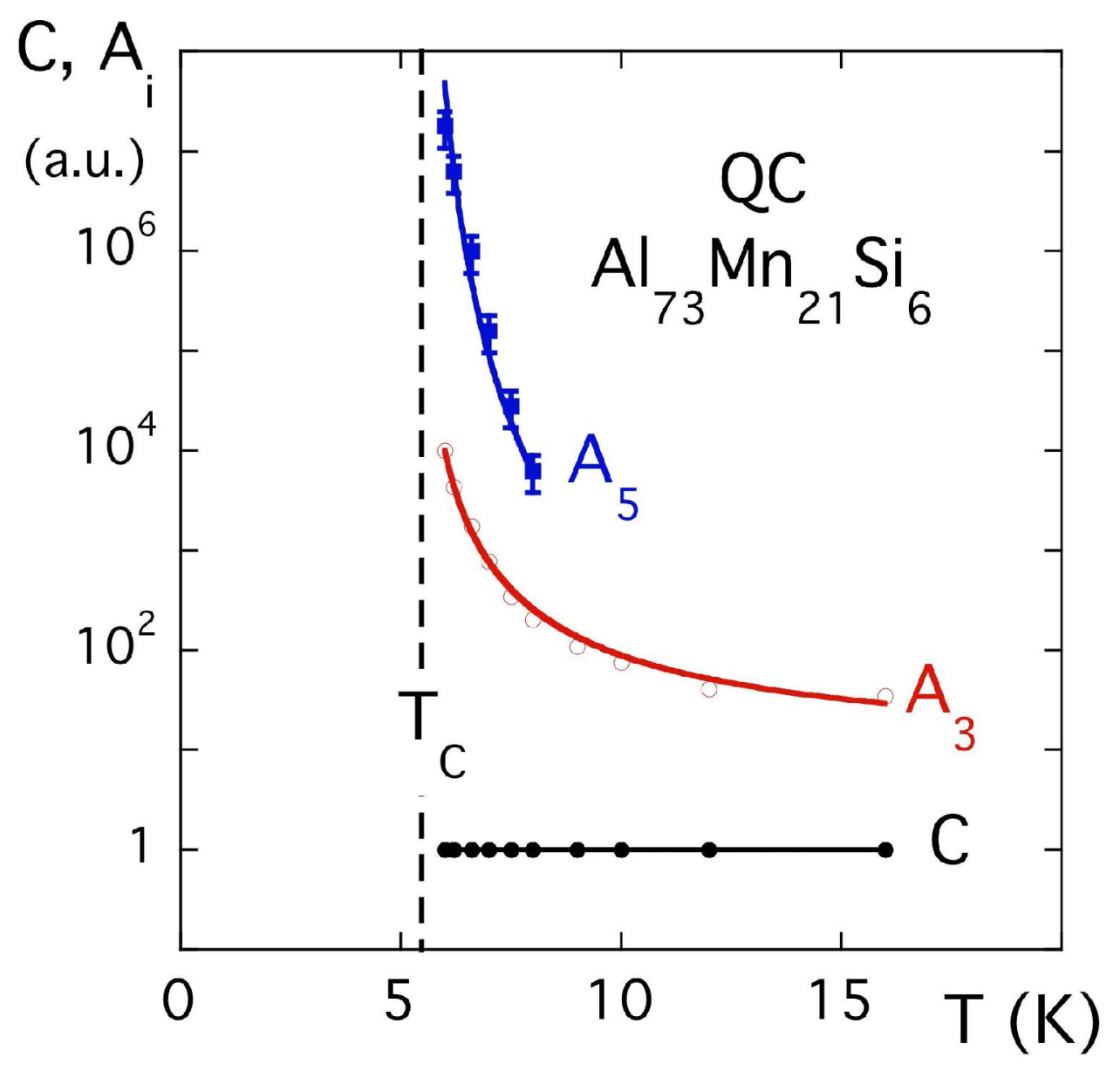

$150 \times 145 \mathrm{~mm}(600 \times 600 \mathrm{DPI})$ 


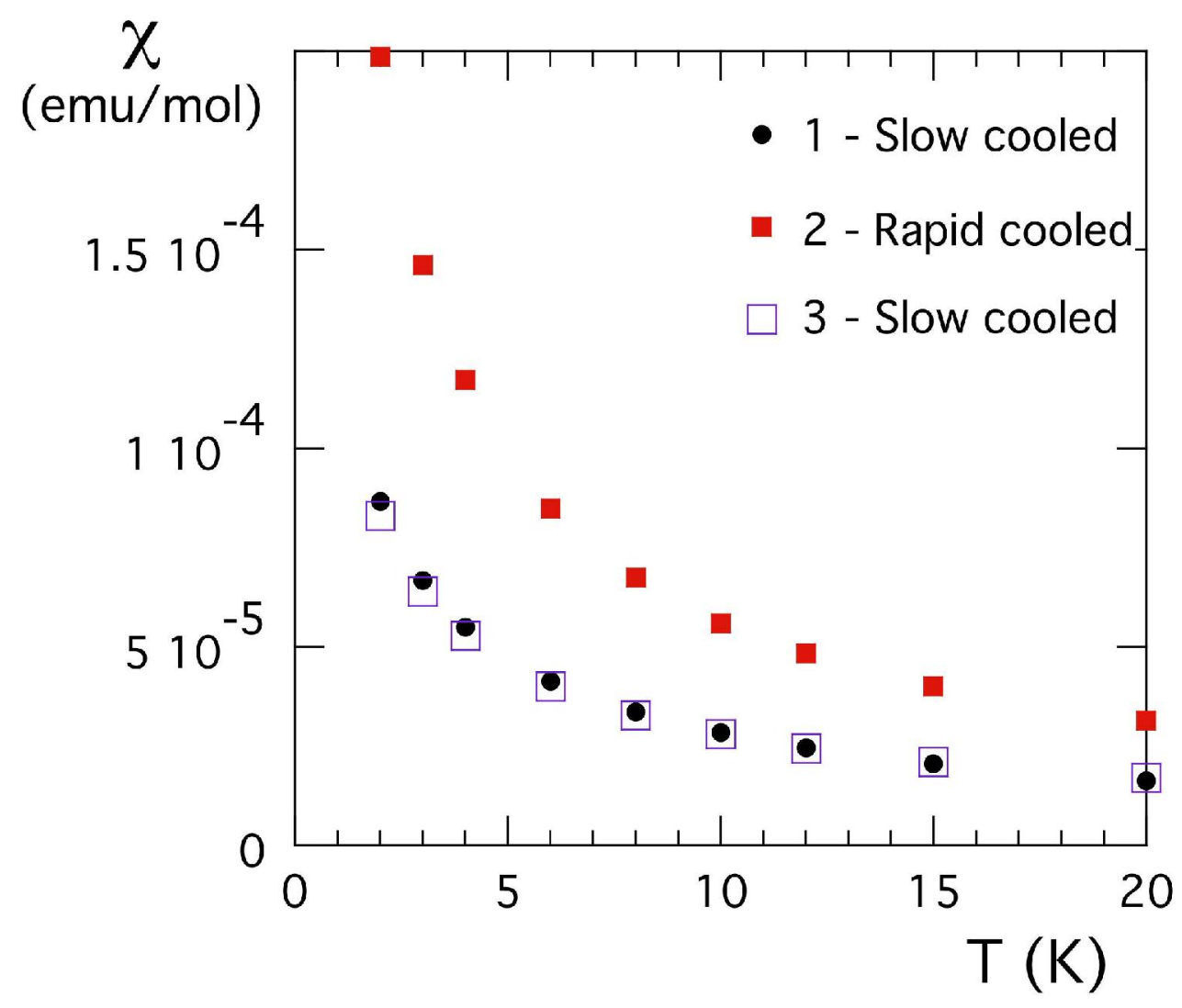

$178 \times 154 \mathrm{~mm}(600 \times 600 \mathrm{DPI})$ 


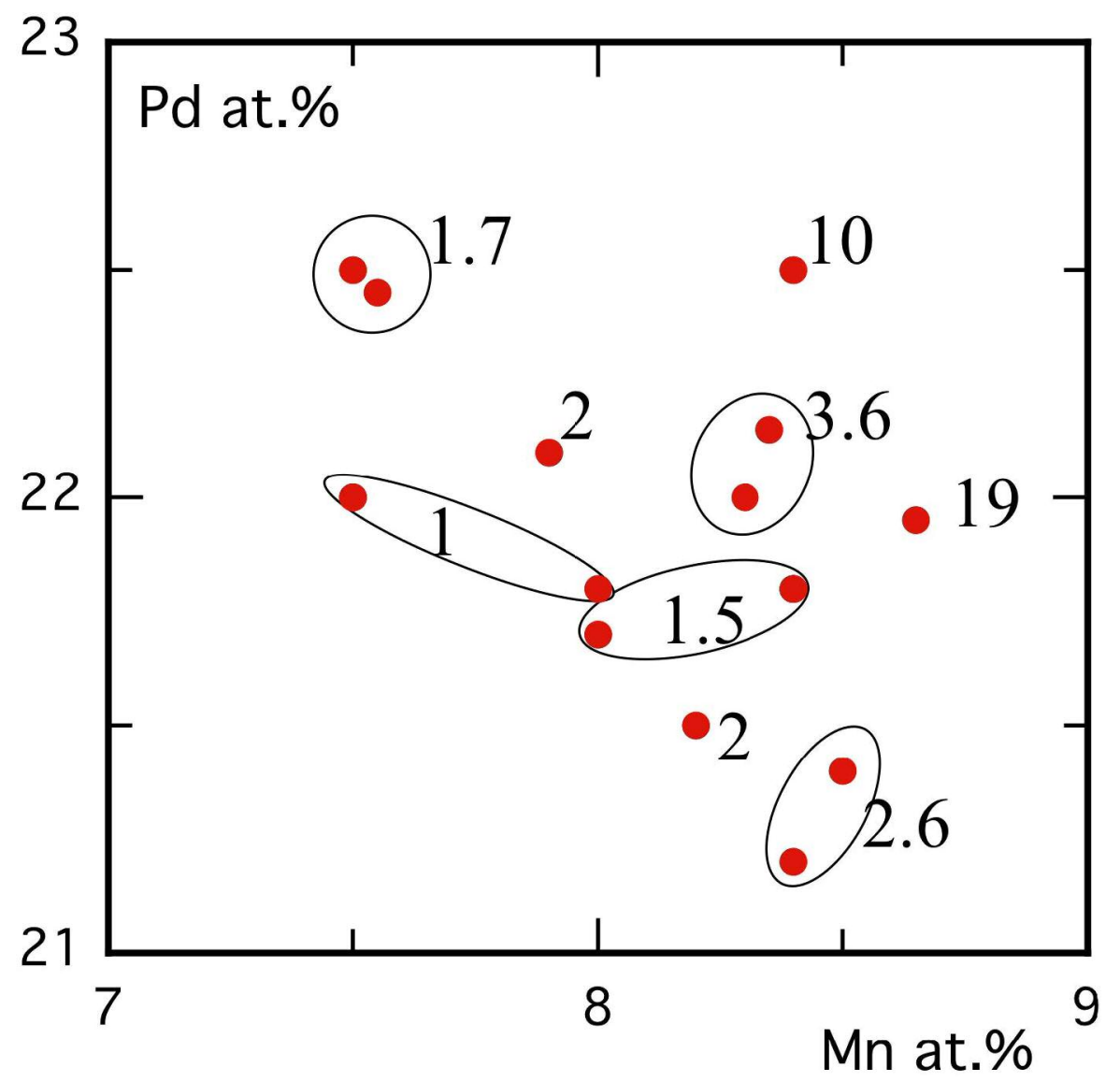

$132 \times 122 \mathrm{~mm}(600 \times 600 \mathrm{DPI})$ 


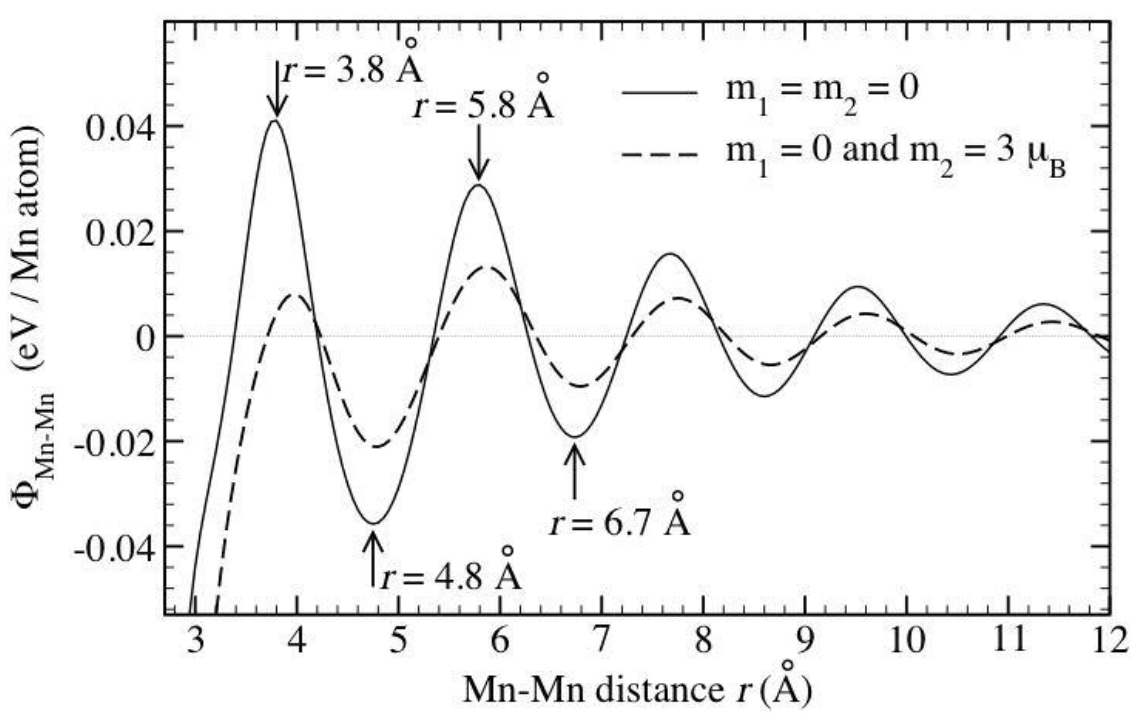

297×209mm (72 x 72 DPI) 
Philosophical Magazine \& Philosophical Magazine Letters

Page 32 of 36

1
2
3
4
5
6
7
8
9
10
11
12
13
14
15
16
17
18
19
20
21
22
23
24
25
26
27
28
29
30
31
32
33
34
35
36
37
38
39
40
41
42
43
44
45
46
47
48
49
50
51
52
53
54
55
56
57
58
59
60

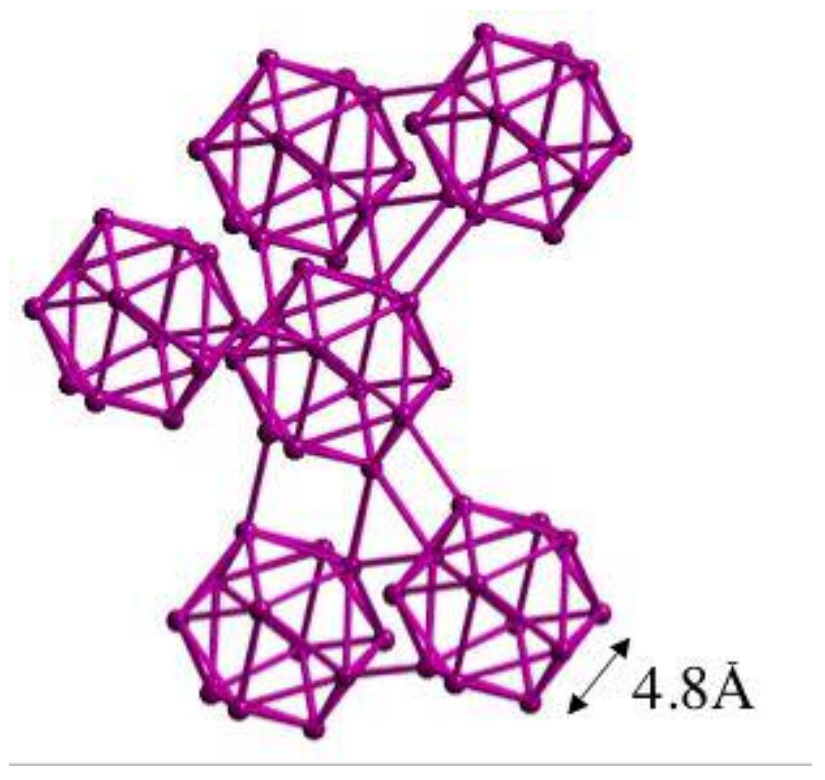

$108 \times 102 \mathrm{~mm}(72 \times 72$ DPI $)$

http://mc.manuscriptcentral.com/pm-pml 


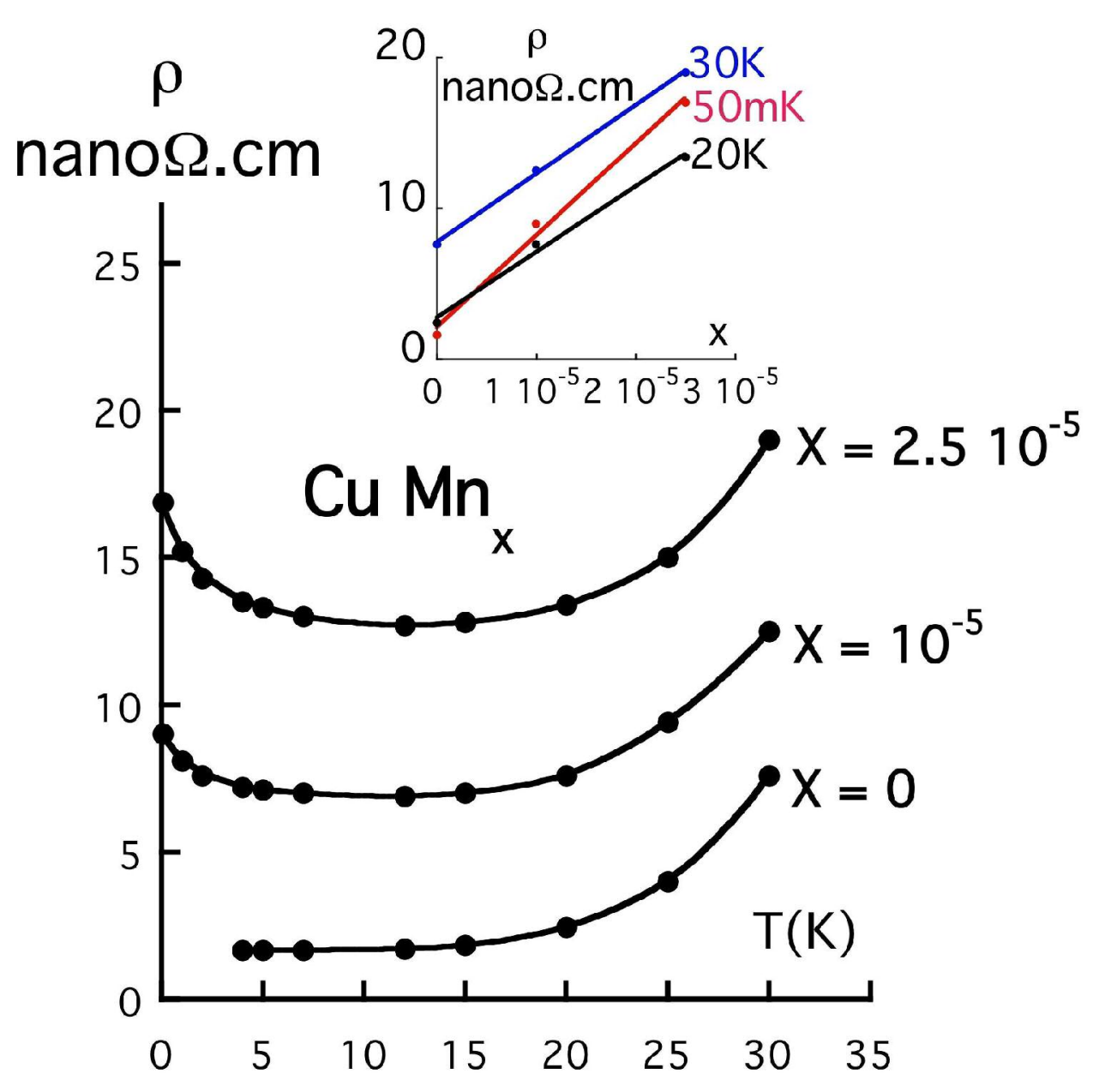

$147 \times 153 \mathrm{~mm}(600 \times 600 \mathrm{DPI})$ 


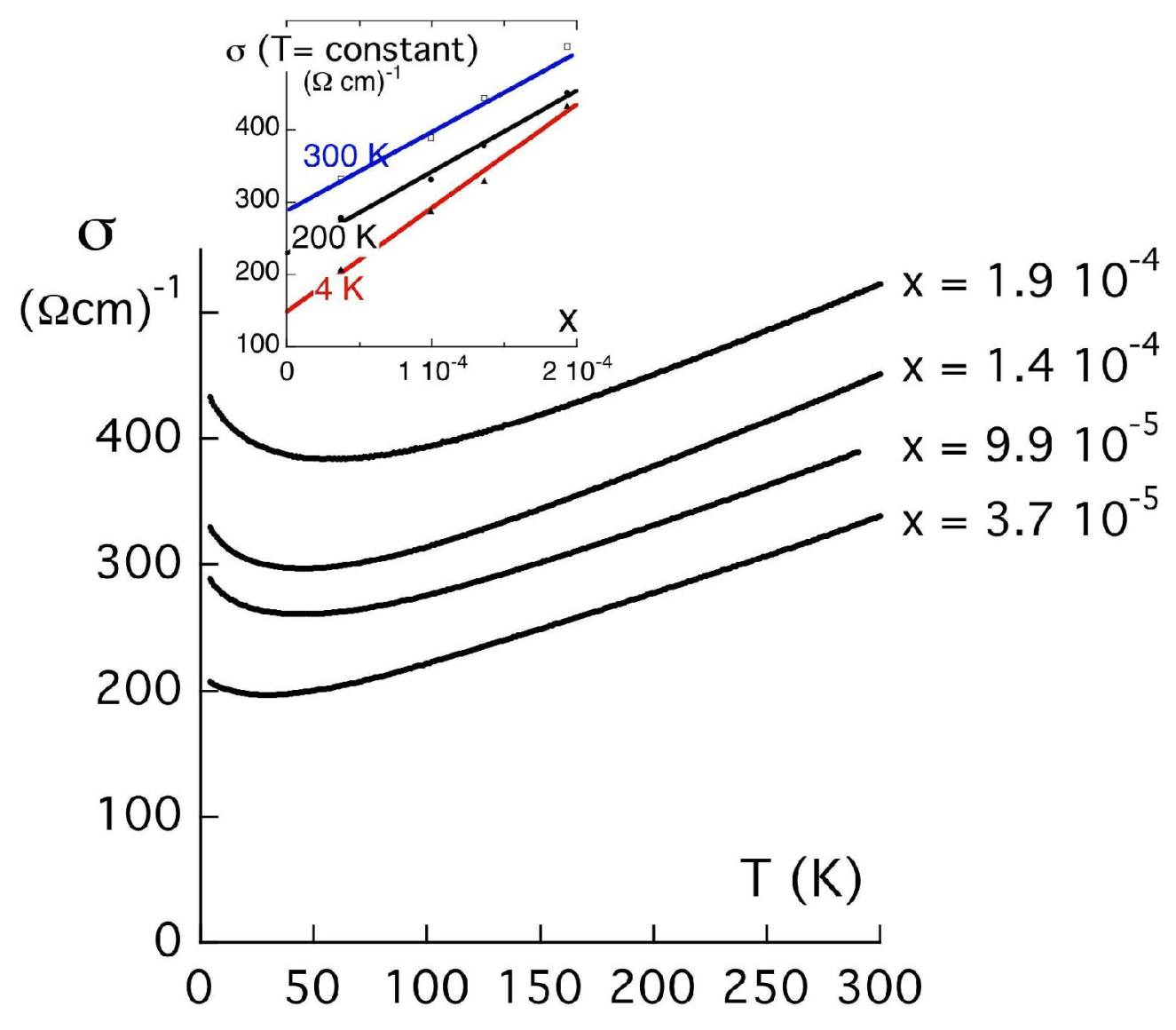

$148 \times 135 \mathrm{~mm}(600 \times 600 \mathrm{DPI})$ 


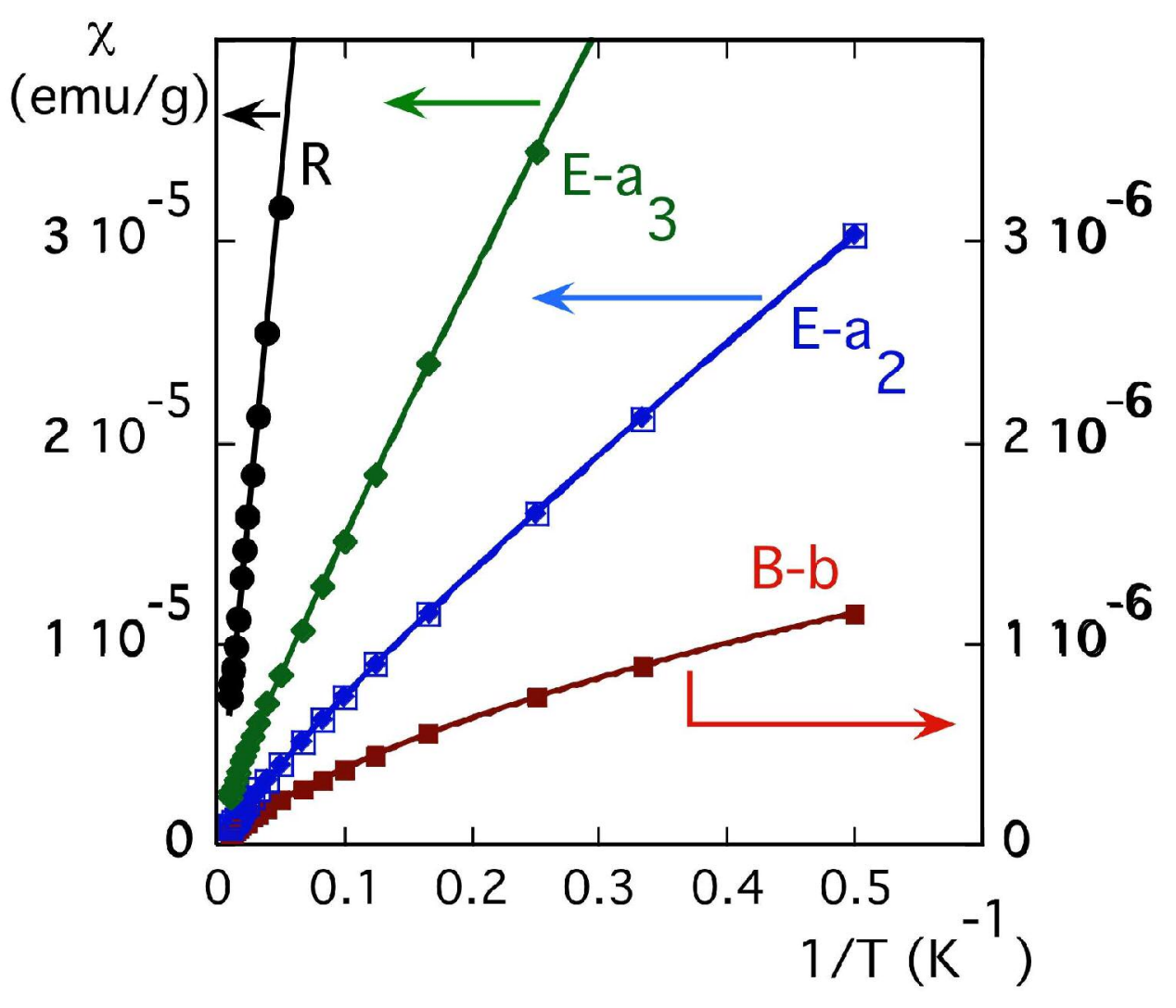

$152 \times 130 \mathrm{~mm}(600 \times 600 \mathrm{DPI})$ 


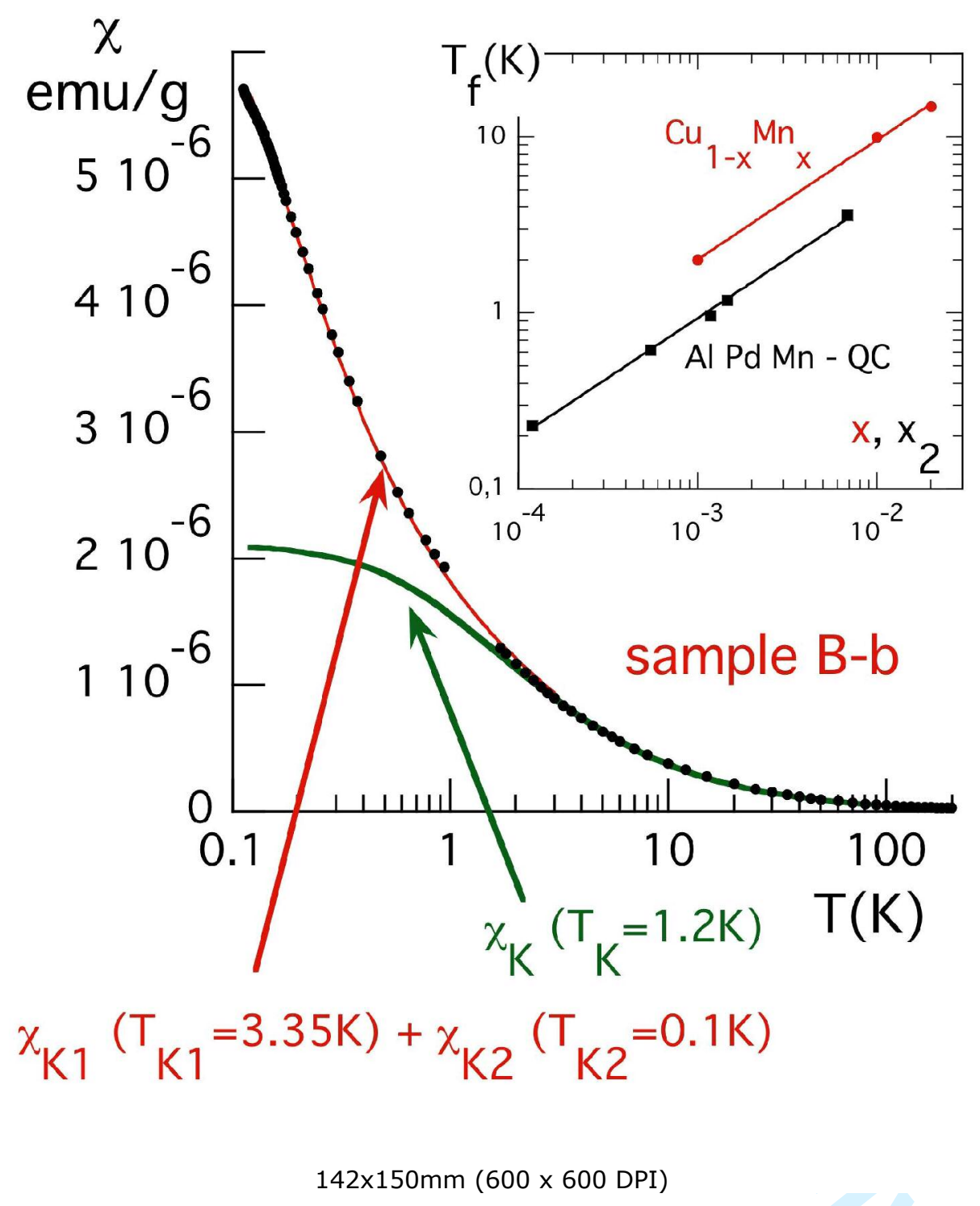

http://mc.manuscriptcentral.com/pm-pml 